\title{
A Comprehensive Overview of the Cold Spot
}

\author{
Patricio Vielva \\ Instituto de Física de Cantabria (CSIC_Universidad de Cantabria), Avenida Los Castros s/n, 39005 Santander, Spain \\ Correspondence should be addressed to Patricio Vielva, vielva@ifca.unican.es
}

Received 27 May 2010; Accepted 17 August 2010

Academic Editor: Dragan Huterer

Copyright (C 2010 Patricio Vielva. This is an open access article distributed under the Creative Commons Attribution License, which permits unrestricted use, distribution, and reproduction in any medium, provided the original work is properly cited.

\begin{abstract}
The report of a significant deviation of the CMB temperature anisotropies distribution from Gaussianity (soon after the public release of the WMAP data in 2003) has become one of the most solid WMAP anomalies. This detection grounds on an excess of the kurtosis of the Spherical Mexican HatWavelet coefficients at scales of around 10 degrees. At these scales, a prominent feature-located in the southern Galactic hemisphere-was highlighted from the rest of the SMHW coefficients: the Cold Spot. This paper presents a comprehensive overview related to the study of the Cold Spot, paying attention to the non-Gaussianity detection methods, the morphological characteristics of the Cold Spot, and the possible sources studied in the literature to explain its nature. Special emphasis is made on the Cold Spot compatibility with a cosmic texture, commenting on future tests that would help to give support or discard this hypothesis.
\end{abstract}

\section{Introduction}

Besides the great success of the NASA WMAP satellite on providing a detailed knowledge of the cosmological parameters that define the physical properties of the Universe (e.g., $[1,2])$, some unexpected results have attracted the attention of the cosmological community soon after the first release of the WMAP data: the so-called WMAP anomalies. Some of these anomalies are related to hemispherical asymmetries (e.g., [3-11]), an anomalous alignment of the quadrupole and octopole components (e.g., [12-21]), significantly low variance of the $\mathrm{CMB}$ temperature fluctuations (e.g., [22$25]$ ), or anomalous alignment of the CMB features toward the Ecliptic poles (e.g., $[26,27])$. Some of these aspects are addressed in this special issue. In addition to the previous findings, the prominent cold spot (hereinafter, the Cold Spot) detected in the southern hemisphere by [28] became one of the most studied anomalies of the WMAP data. The Cold Spot was identified after testing that the Spherical Mexican Hat Wavelet (SMHW) coefficients of the WMAP data presented an excess of kurtosis (at scales of around $10^{\circ}$ in the sky), as compared to the distribution derived from isotropic and Gaussian CMB simulations. This paper presents a complete review on the detection and characterization of this nonstandard signature, and a description of the different attempts made so far in understanding what could be the cause behind such departure from the standard inflationary paradigm. It is organized as follows: in Section 2, the author justifies the use of wavelets as a natural tool for probing the Gaussianity of the CMB temperature fluctuations. the author also presents the different statistics applied in the wavelet space that had led to point out the WMAP data incompatibility with the standard model. In Section 3, the author briefly describes the morphological characteristics of the Cold Spot. The important question of the actual significance of the detection of the Cold Spot and the aspects associated with a posteriori interpretations are addressed in Section 4. Some of the different sources that have been considered in the literature to explain the Cold Spot feature are discussed in Section 5. In Section 6, the author explains in detail a plausible hypothesis to accommodate the existence of the Cold Spot together with the standard cosmological model: a cosmic texture. In addition, the author also describe possible followup tests that could help to confirm or discard such hypothesis. Finally, the author's conclusions are given in Section 7.

\section{The Non-Gaussianity Detection}

The Cold Spot was firstly identified through a blind Gaussianity test of the WMAP first-year data [28]. This test 
was designed to probe the isotropic and Gaussian nature of the $\mathrm{CMB}$, as predicted by the standard inflationary model (see, e.g., [29]), and it was based in a multiresolution analysis performed with the Spherical Mexican Hat Wavelet (SMHW). In this section, the author summarizes the nonGaussianity detection that led to the identification of the Cold Spot. The author starts by justifying why an analysis based on wavelets was proposed and presenting the main characteristics of the wavelet used in the analysis: the SMHW. Afterwards, the author explains which statistics (all of them based on the SMHW coefficients) reported the original deviations from Gaussianity of the WMAP data.

2.1. Why a Wavelet? Nowadays, the CMB scientific community is already very familiar with the application of wavelets (and other members of the -lets zoo, like curvelets, ridglets or needlets) to data analysis. However, it is worth recalling that this is a relatively new custom. Although wavelet applications in cosmology shyly started already in mid-80s, it was not until 1997 that the first application to CMB was presented [30], precisely in an exercise devoted to probe the Gaussianity of the CMB, and it was just a year afterwards that the first $\mathrm{CMB}$ data analyses with wavelets came to light [31, 32], in particular, with two applications to COBE [33] data. During the last decade, the application of wavelets to extract cosmological information from $\mathrm{CMB}$ data has growth considerably, in many different branches: compact source detection (e.g., [34-39]), Gaussianity (e.g., [28, 4051]), cross-correlation with large scale structure (e.g., [5255]), decomposition of the coupled E/B signals [56], probing isotropy (e.g., [9, 26, 27]), cosmic string detection [57], microwave sky recovery (e.g., [58-61]), CMB denoising (e.g., $[34,62]), \mathrm{CMB}$ power-spectrum determination [63], and primordial power-spectrum recovery (e.g., [64]) are some of the application fields. The author refers the reader to $[65,66]$ for some reviews on the wavelets applications to CMB data, with a particular emphasis on data analysis on the sphere.

The wavelet transform (e.g., [67, 68]) has become very popular for a major reason: they offer a unique opportunity to probe scale-dependent phenomena, but keeping, at the same time, information about spatial localization. This is a clear advantage-for many purposes-over classical Fourier or harmonic transforms: physical processes typically exhibit a clear scale-dependent behaviour, and, often, such behaviour differs enough from one phenomenon to another (e.g., in a microwave image, the localized emission due to cluster of galaxies has very different properties as compared to the large-scale signal produced by the Galactic components).

The capability of emphasising or amplifying some features (at a particular scale) makes wavelets unique to probe the Gaussianity of the CMB: there are many different physical processes that might introduce non-Gaussian signatures into a CMB signal, at a very particular scale range (e.g., primordial non-Gaussianity due to nonstandard inflationary scenarios, cosmic defects like strings or textures, secondary anisotropies, foreground emissions, etc.).

In this sense, when a Gaussianity analysis of the CMB is performed in wavelet space, we are in a very adequate

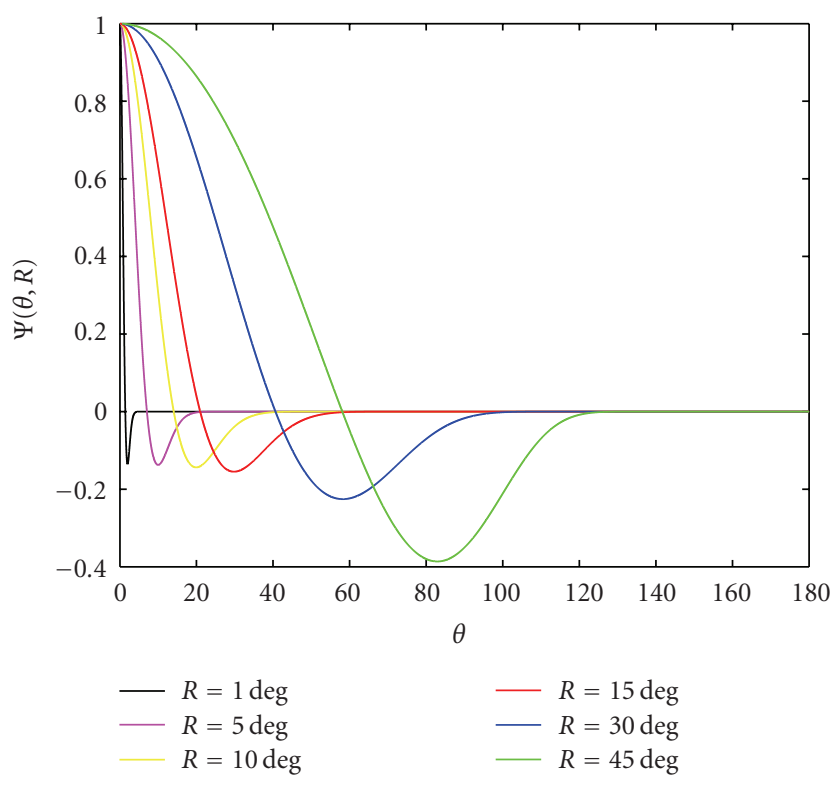

FIGURE 1: Figure shows the profile on the SMHW as a function of the angular distance $\theta$ for different wavelet scales $R$, ranging from $1^{\circ}$ to $45^{\circ}$. Notice that the amplitude of the SMHW at $\theta \equiv 0$ has been fixed to 1 , to allow for an easier comparison.

framework to probe, almost separately, any potential nonGaussian signatures present in the data. The ability of wavelets to amplify a given signature is explained because they can be seen as compensated filters (i.e., $\int_{\Re^{n}} \Psi=0$, where $\mathfrak{R}^{n}$ is the space where the wavelet $\Psi$ is defined). This property is not satisfied by other standard filters, as the tophat or the Gaussian functions. As mentioned above, wavelets do not only provide us with the capability of selecting a given scale range, but they also allow one to keep the information about spatial localization: we do not just study, for instance, the compatibility with Gaussianity, but we are also able to identify where in the data a deviation might be spatially localized. This intrinsic property of the wavelet transform (spatial localization) is also unique to explore not only Gaussianity, but also isotropy, since the statistical properties can be studied (almost independently) from one region in the data to another, in a self-consistent way. Hereinafter, the author will focus on the use of the two-dimensional (2D) continuous wavelet transform (CWT, see, e.g., Chapter 2 in [68]) and, in particular, on the 2D CWT defined on the sphere. There are different ways to define wavelets on the sphere (e.g., [69-72]); most of the works related to the Cold Spot have been performed using the definition of the isotropic Spherical Mexican Hat Wavelet (SMHW) proposed by [45] which is a stereographic projection of the Mexican Hat Wavelet, as proposed by [69]

$$
\Psi(\theta ; R)=\frac{1}{\sqrt{2 \pi} R N_{R}}\left[1+\left(\frac{y}{2}\right)^{2}\right]^{2}\left[2-\left(\frac{y}{R}\right)^{2}\right] e^{-y^{2} / 2 R^{2}},
$$

where $y \equiv 2 \tan (\theta / 2)$ is the stereographic projection variable, $\theta \in[0, \pi)$ is the colatitude, and the constant $N_{R} \equiv$ $\sqrt{1+\left(R^{2} / 2\right)+\left(R^{4} / 4\right)}$ is chosen such as the square of the 
wavelet function $\Psi(\theta ; R)$ is normalized to unity. In Figure 1, the radial profile of the SMHW, for different wavelet scales $R$, is shown. For a signal $T(\theta, \phi)$ defined on the sphere, its spherical harmonic coefficients $t_{\ell m}$ are defined as

$$
t_{\ell m}=\int \mathrm{d} \Omega Y_{\ell m}^{*}(\theta, \phi) T(\theta, \phi),
$$

where $\mathrm{d} \Omega=\mathrm{d} \theta \sin \theta \mathrm{d} \phi$, the spherical coordinates are, as mentioned above, the colatitude $\theta$ (related to the latitude $b$ as $b=\pi / 2-\theta)$, and $\phi \in[0,2 \pi)$ is the longitude. The function $Y_{\ell m}(\theta, \phi)$ is the spherical harmonic of order $\ell$ and $m$, and $*$ denotes complex conjugation. For an isotropic 2D CWT on the sphere, the wavelet coefficients $w(\theta, \phi ; R)$ are obtained as

$$
w(\theta, \phi ; R)=\sum_{\ell=0}^{\ell_{\max }} \sum_{m=-\ell}^{\ell} t_{\ell m} \Psi_{\ell}(R) Y_{\ell m}(\theta, \phi) T(\theta, \phi),
$$

where $\Psi_{\ell}(R)$ is the window function associated with the wavelet function (e.g., $\Psi(\theta ; R)$ in $(1)$ ), and $\ell_{\max }$ represents the maximum multipole associated with a given resolution of the signal $T(\theta, \phi)$, typically limited by the size of the pixel adopted to represent such signal on the sphere.

2.2. The Statistics. The Cold Spot was firstly detected via a positive deviation of the kurtosis of the SMHW coefficients at scales of around $R \approx 300^{\circ}$. The inspection of the map of the SMHW coefficients at these scales revealed the presence of a very large and cold spot in the southern hemisphere. The comparison of the amplitude and the area of this cold spot as compared with Gaussian simulations showed that it was particularly anomalous. Finally, a higher criticism test of the SMHW coefficients also indicated a deviation, at around the same wavelet scales, also showing that the major source for such deviation was located in the position of the Cold Spot. In the following subsections, the author describes briefly these statistics: the kurtosis, the amplitude, the area and the higher criticism.

2.2.1. The Kurtosis. The two most obvious indicators for a possible deviation from Gaussianity of a given data set $z=\left\{z_{1}, z_{2}, \ldots, z_{N}\right\}$ are the third $\left(\mu_{3}\right)$ and fourth $\left(\mu_{4}\right)$ central moments. The $n$-central moment of a random set of $N$ numbers is defined as

$$
\mu_{n}=\frac{1}{N} \sum_{i=1}^{N}\left(z_{i}-\bar{z}\right)^{n},
$$

where $\bar{z}=(1 / N) \sum_{i=1}^{N} z_{i}$ is the mean value of the data set. For a Gaussian random data set of zero mean (i.e., $\mu_{1}=\bar{z} \equiv$ 0 ) and dispersion $\sigma$ (i.e., $\mu_{2} \equiv \sigma^{2}$ ), it is trivial to prove that higher order central moments are either zero (in the case of $\mu_{2 n+1}$, for $n \geq 1$ ) or a given function of the dispersion (in the case of $\mu_{2 n}$, for $n \geq 1$ ). Usually, it is much more convenient to work with normalized central moments $v_{n}$, such as

$$
v_{n}=\frac{\mu_{n}}{\sigma^{n}}
$$

The normalized central moments are more convenient than central moments, since they are referred to the intrinsic fluctuations of the random data set (represented by the dispersion). This helps to absorb into $v_{n}$ possible uncertainties on the knowledge on the amplitude of the fluctuations of the random sample. The normalized central moment $\nu_{3}$ is normally referred to as the skewness $(S)$ whereas $K \equiv \nu_{4}-3$ is called kurtosis. The reason for the subtraction of the number 3 in the previous expression comes from the fact that for a Gaussian random variable, $\mu_{4}=3 \sigma^{4}$ and, therefore, $v_{4}=3$. The previous definition assures that as it happens for the skewness $(S)$, the kurtosis $(K)$ of a Gaussian field is zero. Applying these concepts to the SMHW coefficients $w(\theta, \phi ; R)$, the skewness $S_{R}$ and the kurtosis $K_{R}$ of the wavelet coefficients, as a function of the wavelet scale $R$, can be defined as follows:

$$
\begin{gathered}
S_{R}=\frac{1}{\sigma_{R}^{3}} \frac{1}{N_{\text {pix }}(R)} \sum_{i=1}^{N_{\text {pix }}(R)} w\left(\theta_{i}, \phi_{i} ; R\right)^{3}, \\
K_{R}=\frac{1}{\sigma_{R}^{4}} \frac{1}{N_{\text {pix }}(R)} \sum_{i=1}^{N_{\text {pix }}(R)} w\left(\theta_{i}, \phi_{i} ; R\right)^{4}-3,
\end{gathered}
$$

where at each scale, it is assumed that the coefficients $w(\theta, \phi ; R)$ have zero mean. $\sigma_{R}$ is the dispersion of the wavelet coefficients at the scale $R$

$$
\sigma_{R}=\left[\frac{1}{N_{\text {pix }}(R)} \sum_{i=1}^{N_{\text {pix }}(R)} w\left(\theta_{i}, \phi_{i} ; R\right)^{2}\right]^{1 / 2} .
$$

In the previous expressions, $N_{\text {pix }}(R)$ represents the number of wavelet coefficients at a given scale $R$. Notice that very often, CMB data is not available in the full celestial sphere (e.g., because strong contamination from astrophysical foregrounds has to be masked). In most of the works in the CMB field, the HEALPix tessellation [73] is adopted. In this scheme, the resolution of a given image represented on the sphere is given by the $N_{\text {SIDE }}$ parameter, which indicates how many divisions of the 12 basic pixels are required to achieve such resolution. The $N_{\text {SIDE }}$ parameter is related with the number of the pixels $\left(N_{\text {pix }}\right)$ required to fill the sphere at that resolution as: $N_{\text {pix }}=12 N_{\text {SIDE }}^{2}$. In Figure 2, the major result of the seminal work [28] on the Cold Spot is shown. It represents the kurtosis of the wavelet coefficients, as a function of the scale, for the first release of the WMAP data (blue stars). The solid yellow line represents the mean value obtained from 10,000 CMB Gaussian random simulations, taking into account the instrumental properties of the analyzed data, and generated from an angular power spectrum derived from the best-fit cosmological model. The coloured regions (red, green, and magenta) represent the acceptance intervals at $32 \%, 5 \%$, and $1 \%$, respectively. Notice that at $R$ scales of 250 and $300 \mathrm{arcmin}$, the kurtosis of the WMAP data was above the $1 \%$ acceptance interval. In detail, the excess of kurtosis is given by a $p$-value of $\approx 4 \times 10^{-3}$ for the two scales. No significant deviations were found related to the skewness of the wavelet coefficients. The analysis was repeated in the two Galactic hemispheres separately. This was motivated by previous findings of asymmetries related to the genus [74] and the N-point correlation function [3]. 


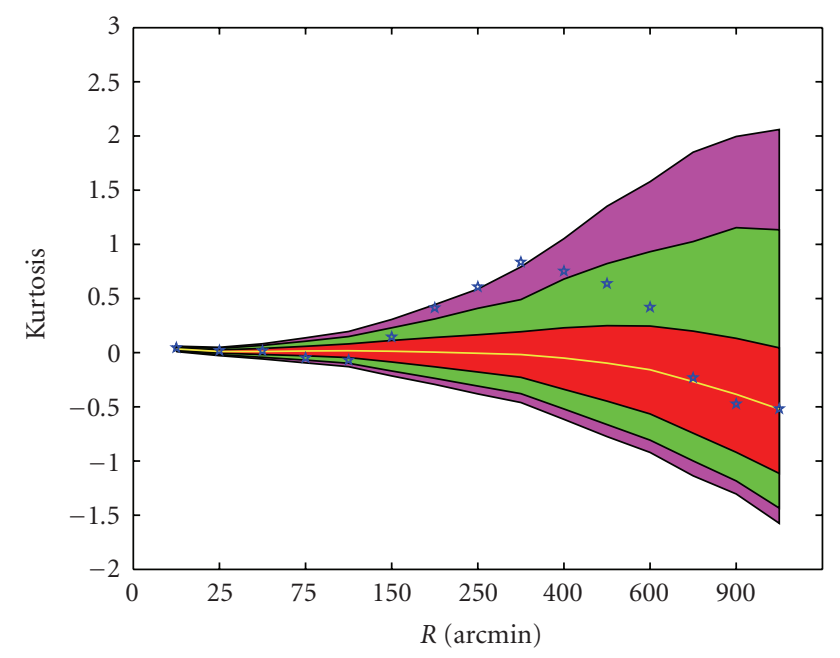

FIGURE 2: Figure showing the positive deviation of the kurtosis of the wavelet coefficients at scales $R$ of 250 and 300 arcmin found by [28]. Red, green and magenta regions represent the acceptance intervals at $32 \%, 5 \%$, and $1 \%$, respectively.

It was found that whereas there was not deviation on the northern hemisphere, the excess of kurtosis was even more remarkable in the southern region. In particular, at the scale of $250 \mathrm{arcmin}$, the kurtosis of the SMHW coefficients was associated with a $p$-value of $\approx 2 \times 10^{-3}$. Again, no deviation on the skewness was noticed. Hence, these analyses indicated that the source for the deviation on the kurtosis of the wavelet coefficient was related to feature/s with a typical length of around $10^{\circ}$ in the sky and located in the southern hemisphere. Thanks to the frequency/spatial properties of the SMHW decomposition, it was possible to study in detail such features. In the left panel of Figure 3, the cleaned CMB map obtained from the fourth release of the WMAP data is given. In particular, the author plot the optimal combination for cosmological analysis (hereinafter, the QVW map), obtained as a noise-weighted linear combination of the Q-, V-, and W-bands, previously cleaned via a template fitting (e.g., [75]). Regions highly contaminated by Galactic foregrounds, nearby clusters, and extragalactic point sources have been masked. In the right panel, the SMHW coefficients of the previous map are represented, at the scale $R=$ $250 \mathrm{arcmin}$. It is evident the presence of the Cold Spot in the southwest side of the image. In particular, the centre of the Cold Spot is estimated to be $\theta=147^{\circ}$ and $\phi=209^{\circ}$. The study of the Cold Spot, through the application of follow-up tests, provided further evidences for its anomalous nature. The author reviews these tests in the following subsections.

2.2.2. The Amplitude. One of the most trivial statistics to study extreme values (as the Cold Spot) in a random sample is the largest/smallest observation. In [28], it was established that the temperature of the Cold Spot was -4.57 times the dispersion of the SMHW coefficients at $R=250 \mathrm{arcmin}$. This cold value represented a $p$-value of .01 (relative to Monte Carlo simulations). A more robust statistic related to the extreme values is the MAX statistic, understood as the largest observation (in absolute value). For the particular case of the SMHW coefficients, MAX is defined, at scale $R$, as

$$
\operatorname{MAX}_{R}=\max \left\{\left|w\left(\theta_{i}, \phi_{i} ; R\right)\right|\right\} .
$$

The MAX statistic is more robust than selecting the coldest of the extrema, since the selection of the lowest values could be seen as an a posteriori selection. This statistic was studied in [76], showing that the Cold Spot was always the maximum absolute observation of the WMAP data at scales around 300 arcmin, representing an upper tail probability of $0.38 \%$ (relative to Monte Carlo simulations). This value was less significant than the one mentioned in the previous subsection. The reason for this change is, as commented, that the MAX statistic is more robust than simply selecting the smallest values of the observations.

2.2.3. The Area. The area above or below a given threshold is one of the most common statistics used to characterize the properties of a random field. In particular, the area is the most commonly Minkowski functional used in the literature (see, e.g., [77-79]). (For 2D images, there are three Minkowski functionals, namely, the contour or length, the area, and the genus. These three quantities are defined above/below a given threshold.) Generalizing this concept to the case of the wavelet coefficients, we can define cold $\left(A_{R}^{-v}\right)$ and hot $\left(A_{R}^{+\nu}\right)$ areas, at a given threshold $\nu$ and a given scale $R$, as

$$
\begin{aligned}
& A_{R}^{-v}=\#\left\{w\left(\theta_{i}, \phi_{i} ; R\right)<-v\right\}, \\
& A_{R}^{+v}=\#\left\{w\left(\theta_{i}, \phi_{i} ; R\right) \geq+\nu\right\},
\end{aligned}
$$

where the number operator $\#\left\{\right.$ condition $\left._{i}\right\}$ indicates how many times condition ${ }_{i}$ is satisfied, for $i$ ranging from 1 to $N_{\text {pix }}(R)$. The cold and hot areas of the WMAP data were analyzed by [46]. It was reported that whereas the hot area was consistent with the expected behaviour for the standard Gaussian model (at all the scales $R$ and thresholds $\nu$ ), the cold area was not compatible. In particular, deviations from Gaussianity were found, again, at SMHW scales of $R \approx 300^{\circ}$. The deviation took place for thresholds equal or smaller than $-3 \sigma_{R}$ (see Figure 4 ). The analysis per different regions of the sky confirmed that the anomaly on the cold area was localized in the southern-west Galactic quadrant of the sky, and that the Cold Spot was responsible for this anomaly. In particular, the cold area of the WMAP data (at the mentioned scales, and below a threshold of $-3 \sigma_{R}$ ) was found anomalous with a probability of $\approx 99.7 \%$ whereas it became fully compatible with Gaussian simulations, once the Cold Spot was not considered in the analysis. As for the case of the MAX statistic, a more conservative estimator (i.e., less dependent from the fact that the Cold Spot is negative) can be considered, just by selecting the maximum value of the previous cold and hot areas

$$
A_{R}^{v}=\max \left\{A_{R}^{-v}, A_{R}^{+\nu}\right\} .
$$

This new statistic was used by [76], finding, again, that the WMAP data was anomalous about thresholds larger than $\left|3 \sigma_{R}\right|$, for scales of the SMHW of around $300 \mathrm{arcmin}$. 


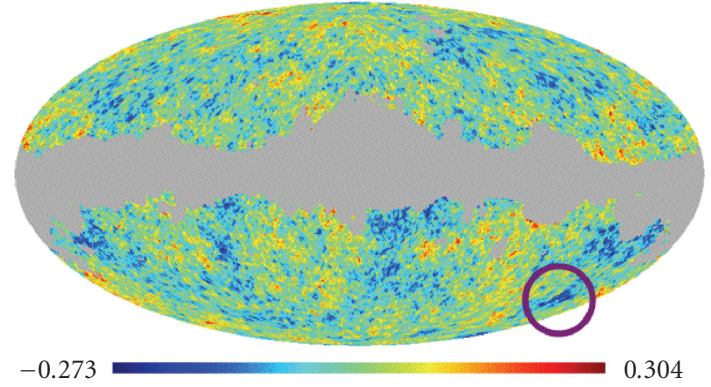

(a)

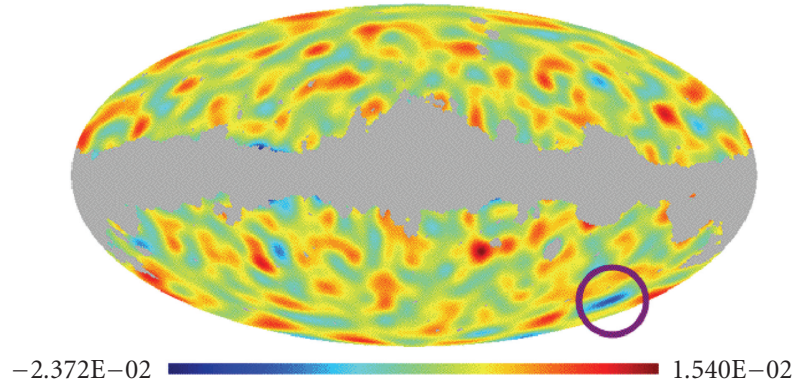

(b)

FIGURE 3: (a) CMB cleaned map derived from the 4th release of the WMAP data, obtained via the template fitting technique described in [75]. (b) wavelet coefficients of the previous map, obtained after the SMHW convolution at a scale of $R=250$ arcmin. The location of the Cold Spot is indicated in both panels by the circle. The centre of the Cold Spot is $\theta=147^{\circ}$ and $\phi=210^{\circ}$.

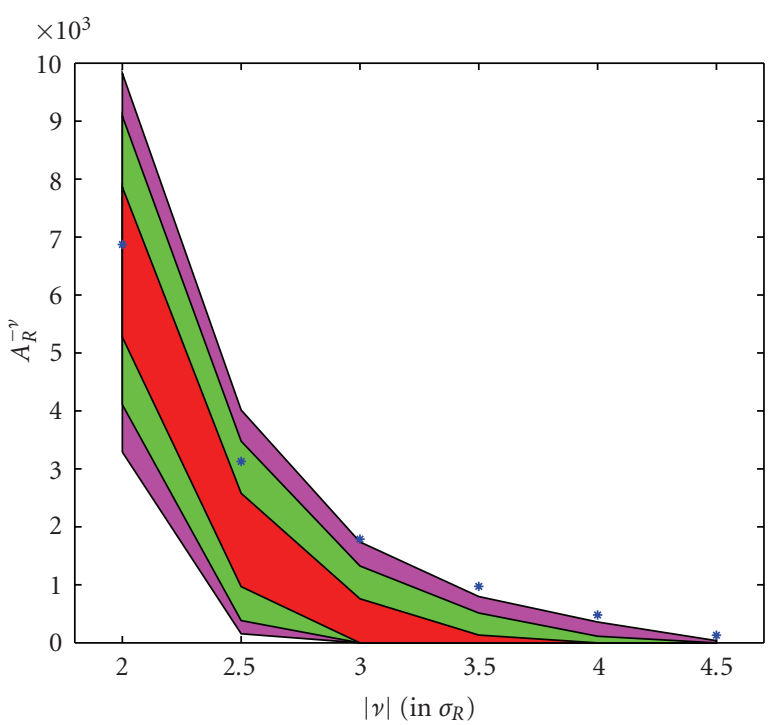

(a)

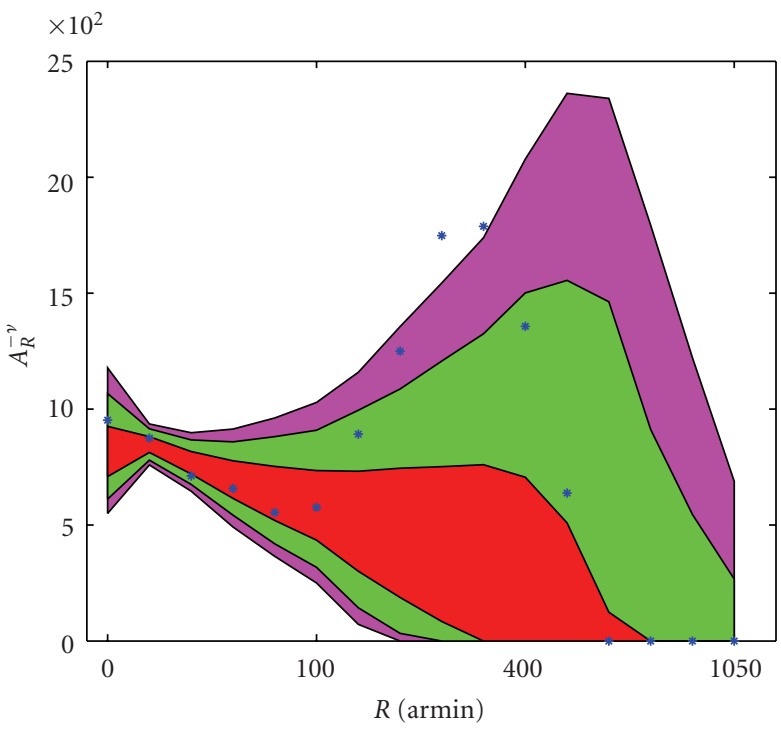

(b)

Figure 4: (a) cold area of the SMHW coefficients (at $R=300 \mathrm{arcmin}$ ), as a function of the threshold $(\nu)$. (b) cold area $\left(A_{R}^{-\nu}\right)$ of the SMHW coefficients (at $\nu=3 \sigma_{R}$ ), as a function of the scale $(R)$. As in Figure 2, the red, green, and magenta regions represent the 32\%, $5 \%$, and $1 \%$ acceptance intervals, respectively. These plots correspond to the analysis done by [46].

2.2.4. The Higher Criticism. Higher criticism (HC) is a relatively new statistic introduced in 2004 by [80], and firstly applied to the context of probing the Gaussianity of the CMB only a year after by [81]. Although there is not a unique definition for the $\mathrm{HC}$, all the forms proposed in the literature satisfy the same key concept: $\mathrm{HC}$ is a measurement of the distance between a given sample of $n$ elements to a Gaussian probability density distribution, established by means of the difference between the $p$-value $p_{i}$ of a given observation $X_{i}$ assuming it comes from a $N(0,1)$ - and its cardinal position on the sorted list (in increasing order) of $p$-values $p_{i}$ (i.e., $p_{i-1}<p_{i}<p_{i+1}$, forall $\left.i=1, \ldots, n\right)$. The HC associated with the sample is just defined as the largest value of such differences.

This concept can be applied to the SMHW coefficients of a given signal (e.g., the QVW map) at a given scale $R$. This was the analysis proposed by [81]. Let us adopt the following definition for the $\mathrm{HC}$ associated with $N_{\text {pix }}(R)$ wavelet coefficients $w\left(\theta_{i}, \phi_{i} ; R\right)$, at scale $R$ :

$$
\mathrm{HC}_{N_{\text {pix }}}(R)=\max \left\{\mathrm{HC}_{N_{\text {pix }}}^{i}(R)\right\}
$$

where the maximization is made over the quantity $\mathrm{HC}_{N_{\text {pix }}}^{i}(R)$, that provides the difference between the experimental probability of the wavelet coefficients $w\left(\theta_{i}, \phi_{i} ; R\right)$ at scale $R$ and the corresponding theoretical pvalue. Such quantity reads as

$$
\mathrm{HC}_{N_{\text {pix }}}^{i}(R)=\sqrt{N_{\text {pix }}} \frac{\left|\left(i / N_{\text {pix }}(R)\right)-p_{i}(R)\right|}{\sqrt{p_{i}(R)\left(1-p_{i}(R)\right)}},
$$

where the $p$-value is given by $p_{i}(R)=P\{|N(0,1)|>$ $\left.\left|\widehat{w}\left(\theta_{i}, \phi_{i} ; R\right)\right|\right\}$. The ${ }^{\wedge}$ operator indicates that the $N_{\text {pix }}(R)$ SMHW coefficients at the scale $R$ have been transformed into 


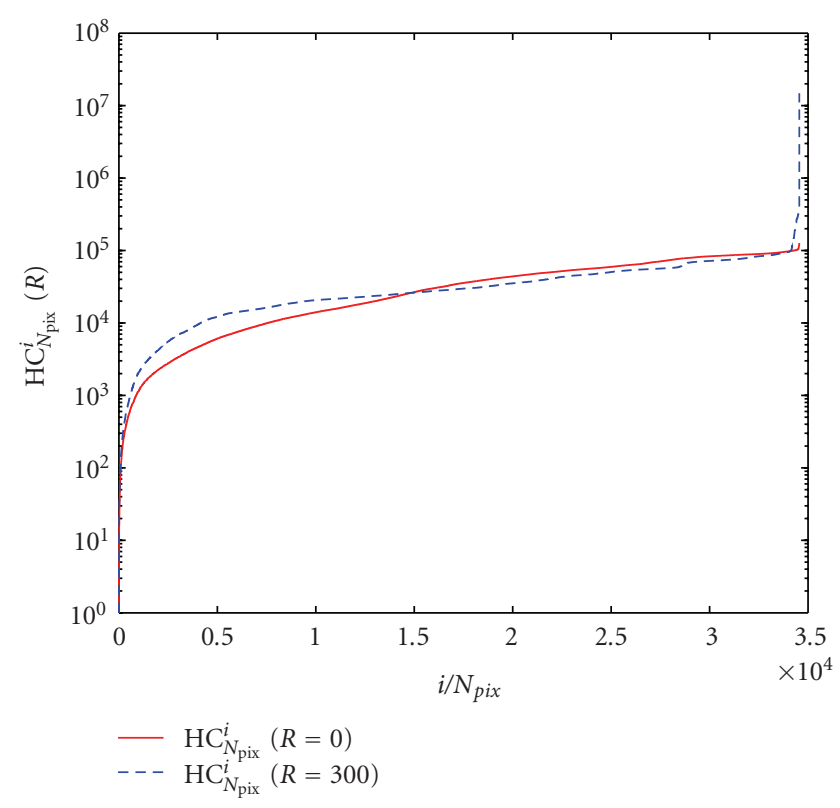

FIGURE 5: The $\mathrm{HC}_{N_{\text {pix }}}^{i}$ values obtained from the analysis of the QVW map. The solid red line corresponds to the application of (12) to the $\mathrm{CMB}$ map in the real space (i.e., $R \equiv 0$ ) whereas the dot-dash blue line corresponds to the analysis of the SMHW coefficients at scale $R=300$ arcmin. $\mathrm{HC}_{N_{\text {pix }}}^{i}$ curves are normalized by their respective minimum values.

a zero mean and unit variance sample. The author remarks that the $p$-values have been sorted in increasing order.

As explained in [81], the $\mathrm{HC}$ represents, under certain conditions, some advantages with respect to more traditional statistics designed to study the Gaussianity of a given sample. In particular, $\mathrm{HC}$ seems to be a better estimator than the MAX statistic: whereas the latter is designed to capture Gaussian deviations caused by very large values of the distribution, the $\mathrm{HC}$ is also sensitive to anomalies produced by moderate values. In addition, the $\mathrm{HC}$ can identify which values (in a given sample) are the ones that differ from the theoretical Gaussian distribution.

In [81], it was reported that the $\mathrm{HC}_{N_{\text {pix }}}(R)$ was above the $1 \%$ acceptance interval, again, at the SMHW scale $R=300$ arcmin. They found that, actually, all the SMHW coefficients associated with $\mathrm{HC}_{N_{\text {pix }}}^{i}(R)$ values above the $1 \%$ acceptance interval set by $\mathrm{CMB}$ Gaussian simulations, where localized in the position of the Cold Spot. This extra test was an additional support to the anomalous nature of the WMAP data, and of the Cold Spot in particular. Results were confirmed by [76] for the analysis of the second WMAP data release, reporting an upper tail probability even lower than for the 1-year data.

As an illustration of the HC statistic, in Figure 5, the author represents (in solid red) the $\mathrm{HC}_{N_{\text {pix }}}^{i}$ values obtained from the QVW map in the real space, and (in dot-dash blue) the corresponding curve for $R=300 \mathrm{arcmin}$. These quantities are normalized to their minimum values, for a better comparison. They are represented in an increasing order. It is remarkable that, for the case of the analyses performed on the SMHW coefficients, there is a tail of very large values of $\mathrm{HC}_{N_{\mathrm{pix}}}^{i}$, that are not present for the real space case. This is due to the ability of the SMHW transform to enhance features of a given scale and shape. In Figure 6, these values are represented on the celestial sphere (left panel for the real space case, and right panel for the SMHW coefficients). The figure indicates that there are not particular signatures in the real space whereas the SMHW coefficients at $R=300$ arcmin allow us for a clear identification of the features causing the anomalous values of the $\mathrm{HC}_{N_{\text {pix }}}(R)$. In particular, the key role played by the Cold Spot is highlighted.

\section{The Characteristics of the Cold Spot}

In this section, the author summarizes briefly some of the most important properties of the Cold Spot. The author will focus in two major aspects: its morphology and its frequency dependence.

The morphological properties of the Cold Spot are different depending whether we do the analysis in the real or wavelet space. As it was pointed out in [46], the region associated with the Cold Spot, in the real space, appears as formed by several small cold spots. The amplitude of the most prominent of these spots is $\lesssim-350 \mu \mathrm{K}$ with a size of $\approx 1^{\circ}$. None of these structures is particularly anomalous. The image of the Cold Spot in the real space is shown in the left panel of Figure 7. It is, however, in the wavelet space where the Cold Spot appears more interesting. In the right panel of Figure 7, the author presents a close view of the Cold Spot after convolution with the SMHW at a scale $R=250$ arcmin. Besides all the anomalous characteristics previously discussed (i.e., area, HC, and MAX), the Cold Spot appears as a very symmetric feature. However, this effect could be biased since, after all, the SMHW is an isotropic filter and, therefore, the symmetric features of the Cold Spot could be amplified, erasing any possible intrinsic anisotropy. This issue was studied in detail by [82]. Instead of applying an isotropic wavelet, the anisotropic Elliptical Spherical Mexican Hat Wavelet (ESMHW) was adopted. The cleaned CMB map derived from the WMAP was transformed into ESMHW coefficients (at the scales for which the WMAP data appeared as anomalous), for different ratios $\zeta$ between the smallest and the largest axes of the ESMHW, and for different orientations. This work proved that the maximum matching between the Cold Spot and the ESMHW took place when $\zeta \in[0.875,1]$ and, hence, indicated that the Cold Spot structure was quite close to be isotropic (assuming that the ratio of the ESMHW axes mimics, somehow, the ratio between the Cold Spot axes).

The frequency dependence of the Cold Spot has been a matter of study soon after its discovery. Already in [28], the SMHW coefficients of the cleaned WMAP frequency channels (namely, $Q, V$, and $W$ bands) were computed, and the mean value of the coefficients associated with the Cold Spot at the scale $R=250 \operatorname{arcmin}$ was estimated. No obvious frequency dependence of this mean value of the wavelet coefficients was found, hence, being fully consistent with the expected behaviour for the CMB emission and, 


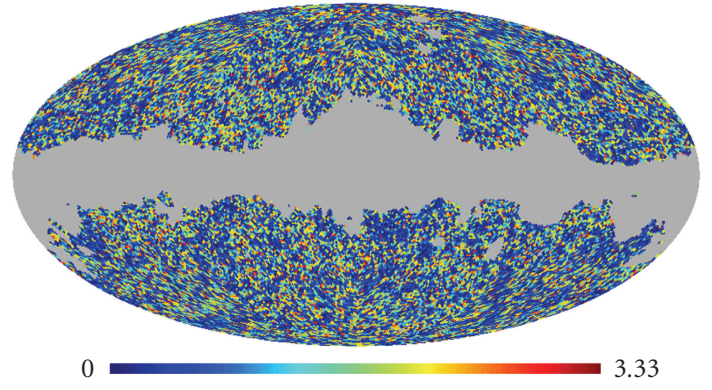

(a)

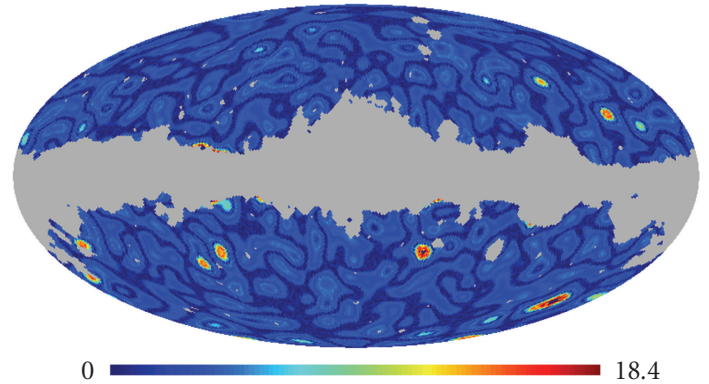

(b)

FIGURE 6: Maps of $\mathrm{HC}_{N_{\text {pix }}}^{i}$ obtained from the analysis of the QVW map derived from the WMAP data. Left panel corresponds to the study of the real space case, while right panel shows the outcome of the analysis of the SMHW coefficients at $R=300$ arcmin. Whereas for the former there are not particular signatures in the map, the wavelet analysis shows some prominent features, being the Cold Spot the most pronounced one.

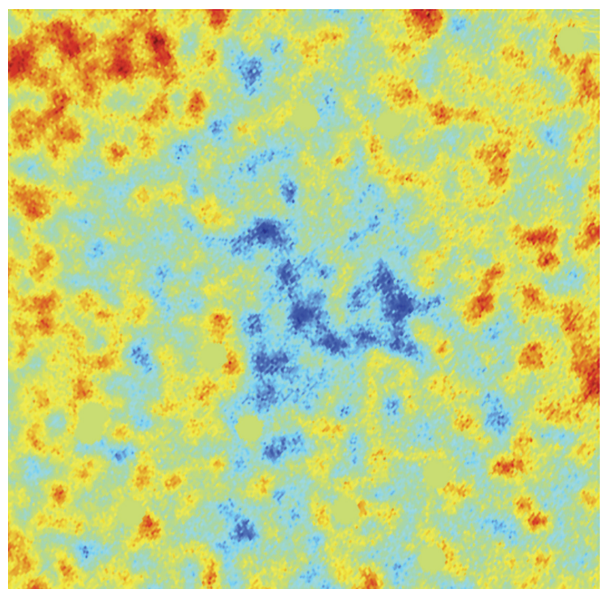

(a)

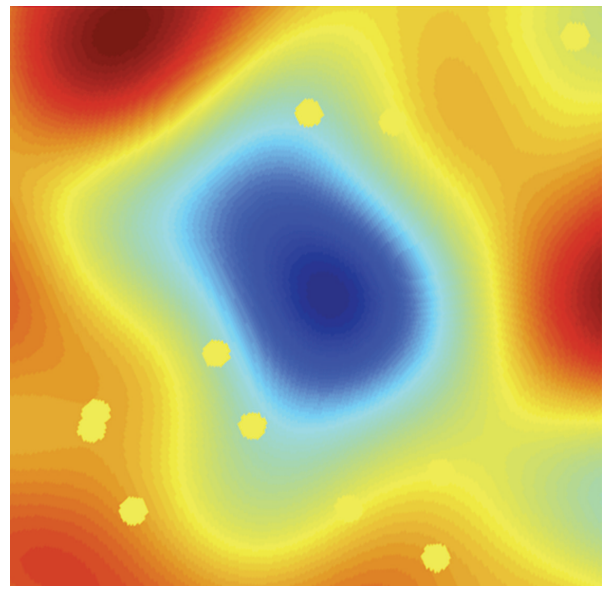

(b)

FIGURE 7: Image of the Cold Spot $\left(\theta=-147^{\circ}, \phi=209^{\circ}\right)$ in the real (a) and wavelet (b) spaces. The homogenously filled circles correspond to positions where known extragalactic point sources have been masked.

therefore, quite different to the typical frequency dependence of the Galactic foregrounds. (The analyzed WMAP frequency channels were in thermodynamical temperature and, therefore, the $\mathrm{CMB}$ appears as a frequency independent emitter. Notice that, since the SMHW transform is a linear operation, the same behaviour is expected for the wavelet coefficients.)

\section{The Significance of the Detection: The A Posteriori Issue}

One of the most questioned aspects of the WMAP anomalies in general, and of the Cold Spot in particular, is the issue of the actual significance of the detection. This is a very important point that is intimately linked to the blind nature of all the Gaussianity/isotropy tests that led to the report of such anomalies.

The author reviews where this problem comes from: if many tests are performed in a given data set, it is not strange that some of them report some deviation from the null hypothesis. It is quite usual to face the following situation: a set of blind tests (i.e., tests that just challenge the compatibility of the data with a given null hypothesis, $H_{0}$, and not confronting such hypothesis with an alternative one, $H_{1}$ ) claim a given incompatibility of the WMAP data. A subsequent test is performed, taking into account the previous finding and, usually, in such a way that the initial reported deviation is now found at higher significance. In this procedure, there are two weak points: the first one, already mentioned, is to assess the probability of finding a deviation as the one claimed during the first step, taking into account all the possible tests that were performed. The second one is the credibility of the probability for the followup test, where a particularity was studied in greater detail.

As mentioned above, this is a common situation for the WMAP anomalies works and, therefore, the Cold Spot is not an exception. Several tests were made in the first work by [28], namely, the estimation of the skewness and the kurtosis at several scales of the SMHW. A particular deviation was highlighted: the excess of kurtosis at several scales around $R=250$ arcmin. After that, the Cold Spot was identified as a prominent feature, and further tests (the MAX, the cold 
area, the HC) were applied. The author believes that there is not a unique and clear way to solve these ambiguities and, to the author's view, this point is not usually addressed in the literature. However, whereas for the latter aspect (i.e., the significance for the follow-up tests) the solution is hard, for the former there could be some possible getaway, at least depending on the complexity of the preliminary analysis. Actually, the Cold Spot is one of the few WMAP anomalies where this particular aspect has been considered with deeper interest. In fact, it was the matter of several papers $[47,82]$ and, in particular, of [76].

In this last work, the significance of the first detection was addressed, focusing in the a posteriori selection of the statistic (the kurtosis) and the scale range ( $\approx 250 \mathrm{arcmin})$. A conservative procedure to establish the $p$-value of the non-Gaussian detection, based on the characteristics of the analysis, was proposed. More specifically, since 30 statistics were applied to the QVW map (i.e., the skewness and the kurtosis of the SMHW coefficients at 15 scales), and only 3 out of these 30 statistics were found as anomalous (i.e., the kurtosis of the SMHW at scales $R=200,250$, and 300 arcmin were outside the $1 \%$ acceptance intervalsee [76] for details), then, it was decided to estimate the significance of the non-Gaussianity detection by exploring in how many out of 10,000 CMB Gaussian simulations it was observed that the skewness or the kurtosis of the SMHW coefficients were outside the $1 \%$ acceptance interval, at least, at three scales. The $p$-value obtained in this manner was 0.0185 . This $p$-value can serve, as explained in [76], as a conservative probability related to the non-Gaussianity associated with the SMHW analysis.

In this spirit, the following up tests (e.g., the amplitude, the area, or the HC) can be just seen as additional probes to explore/understand the previous deviation, rather than as independent sources for establishing a proper significance level for the detection.

Recently, [83] has questioned the non-Gaussianity found by [28], since the excess of the kurtosis was clearly found with the SMHW, but it was not the case with other analyzing kernels (proposed in [83]), as the top-hat and the Gaussian filters. The authors argued that these tools are more natural than a wavelet like the SMHW and that, therefore, the selection of the SMHW is somehow a posteriori. Contrary to this reasoning, the author found that the results obtained by [83] imply a different conclusion: the lack of detection when analyzing with the top-hat and the Gaussian filter is a proof of the issue discussed in Section 2.1, namely, that any filtering kernel is not necessary suitable for the detection of any non-Gaussian feature. It is clear that some features (like point sources, cosmic strings, or textures) are much better detected after applying optimal or targeted filters, rather than general ones (like the top-hat or the Gaussian functions).

The reason why a compensate filter as the SMHW gets a much larger amplification as compared to uncompensated kernels as the previous ones is that it is much more efficient to remove the background fluctuations above and below a given scale interval. Even more, it can be shown (e.g., [84]) that the SMHW is close to the optimal or Matched filter

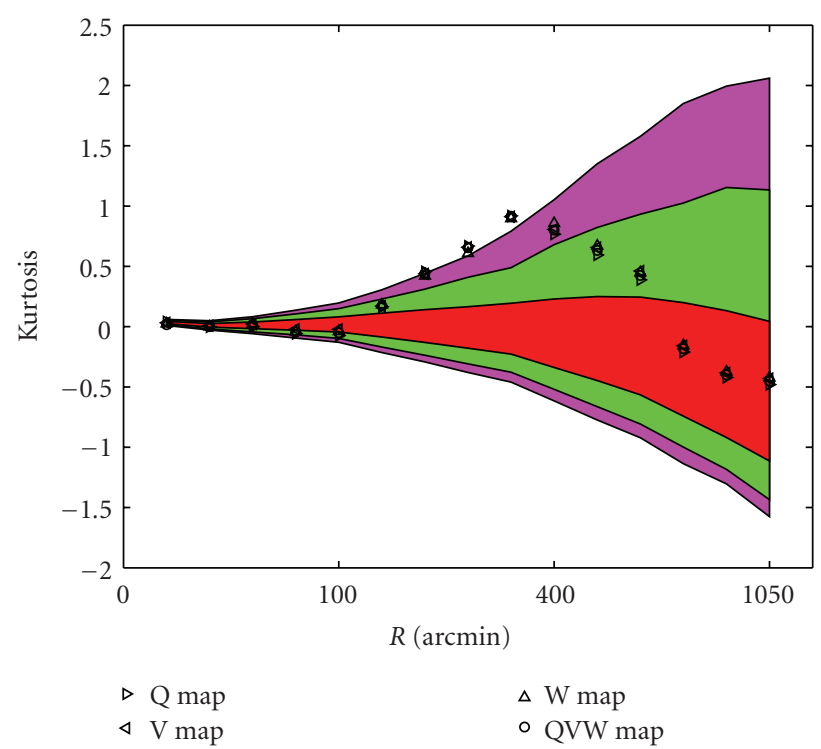

FIGURE 8: Frequency dependence of the excess of the kurtosis of the SMHW coefficients. The plot shows the variation of the kurtosis as a function of the scale $K_{R}$ for cleaned CMB maps obtained from the WMAP data at three cosmological frequencies (Q-, V-, and Wbands), and for the optimal CMB cleaned map provided by WMAP, as noise-weighted combinations of the previous maps (the QVW map). The coloured regions represent, as for other figures, the $32 \%$, $5 \%$ and $1 \%$ acceptance intervals provided by simulations.

to detect objects with a Gaussian-like profile embedded in CMB-dominated background (as it is the case in the region of the Cold Spot). The form of a matched filter designed for a given situation is defined, not only by the shape of the feature to be detected, but also by the statistical properties of the background. In particular, the matched filter (defined in the Harmonic space) is proportional to the shape of the feature and inversely proportional to the angular power spectrum of the background. Therefore, bearing in mind that (at degree scales) the CMB is well described by an angular power spectrum close to $C_{\ell} \propto \ell^{-2}$, it is trivial to show that the SMHW is near to be an optimal tool for detecting a feature described by a Gaussian-like profile and embedded in such background.

The author remarks that as it was said in Section 2.1, the selection of wavelets as a suitable tool for non-Gaussian analysis, as it was shown by several authors in the past (e.g., $[40,45,85])$, cannot be considered as an a posteriori choice, but, rather, as a natural option for studying scale-dependent phomena. In particular, the compensation property satisfied by wavelets makes them extremely good analyzing kernels to amplify certain features, precisely because it assures a strong suppression of the large-scale fluctuations of the background.

Finally, it is worth recalling that the Cold Spot has been identified as an anomalous feature by other tools different from the SMHW: by directional wavelets [47], scalar indices [86, 87], steerable wavelets [27], needlets [48], and the Kolmogorov stochastic parameter [88]. 


\section{Some Possible Sources to Explain the Cold Spot}

To find an explanation for the non-Gaussianity deviation associated with the Cold Spot is the next step, once its anomalous nature (i.e., noncompatible with the standard inflationary scenario) is accepted. With this aim, many efforts have been done in the last years, considering different sources for the observed anomaly. The possible causes addressed so far account for systematics effects, mostly due to instrumental aspects that are not well understood/modelled, such as spurious emissions due to foregrounds or contaminants of the cosmological signal, nonaccounted secondary anisotropies induced on the $\mathrm{CMB}$ photons, as the interaction with the ionized medium (e.g., the Sunyaev-Zeldovich effect) or the nonlinear evolution of the gravitational potential (e.g., the Rees-Sciama effect), and, of course, alternative (or complementary) models to the standard inflationary scenario (as cosmic defects). In the following subsection, and also in Section 6, the author addresses these possibilities, starting from those hypotheses that are less dramatic from the point of view of strong implications for the standard cosmological model.

5.1. Systematics. To test the influence of unknown/unmodeled systematics on the non-Gaussianity deviation is, as one could imagine, a very hard task. The analyses that can be carried out to probe such sources are, basically of two types.

One of these analyses are consistency tests. As in most of the CMB experiments, WMAP satellite can provided us with cleaned $\mathrm{CMB}$ maps (e.g., following the template fitting approach described by [75]) for several detectors. Therefore, focusing in the non-Gaussian deviation associated with the Cold Spot, an obvious procedure would be to check whether the application of the different statistical tools reveals that such a feature is associated with only one detector, or a smaller set of detectors. If this were the case that would be a clear indication for a lack of consistency and, therefore, that the non-Gaussianity detection is associated with a given instrumental feature. This was done by $[28,46]$ for the kurtosis $\left(K_{R}\right)$ and the area $\left(A_{R}^{v}\right)$ of the SMHW wavelet coefficients. No inconsistency was found: the excess of kurtosis and of area was found to be the same for every difference assembly. As an illustration, in 8 , the kurtosis of the SMHW coefficients (as a function of the scale $K_{R}$ ) for 4 different $\mathrm{CMB}$ maps is presented. In particular, results for the Q-, V-, and W-band cleaned CMB maps are shown, together with the curve obtained from the analysis of the optimal QVW-map. These curves are quite similar, which indicates that the non-Gaussian signal is presented in all the WMAP detectors, at a similar level.

The second type of analyses are null tests. The cosmological frequencies of the WMAP satellite (i.e., Q-band at $41 \mathrm{GHz}, \mathrm{V}$-band at $61 \mathrm{GHz}$, and $\mathrm{W}$-band at $94 \mathrm{GHz}$ ) are made from more than one difference assembly. Hence, just subtracting difference assemblies at the corresponding band can produce noise maps per frequency. Neglecting small differences from the optical beams and the band-pass widths, the $\mathrm{CMB}$ and foreground emissions have been cancelled out in this new map. Therefore, the application of the statistical tools to these difference maps helps to check whether the non-Gaussian signal is a noisy artifact (if such signal is still present) or not (if consistency with Monte Carlo simulations is found). These was done by $[28,46]$, again, for the kurtosis and the area of the SMHW coefficients, respectively. As for the previous type of systematics probe, there was a clear indication that the non-Gaussian signal was not related to any instrumental signature. As an example, in Figure 9, the result obtained by [28] for $K_{R}$ is presented. On the left panel, the author represents the variation of the SMHW wavelet coefficients for the difference map constructed at $41 \mathrm{GHz}$ as Q1-Q2. Similarly, the results for the $V 1-V 2$ map at $61 \mathrm{GHz}$ are provided in the middle panel. Finally, in the right panel, the author gives the output for the difference map obtained at the $94 \mathrm{GHz}$ band as the combination $W 1-W 2+W 3-$ $W 4$. Results for the WMAP data is given as blue asterisks whereas, as for previous figures, the red, green, and magenta regions provide the $32 \%, 5 \%$, and $1 \%$ acceptance intervals, respectively.

Summarizing, consitency and null tests do not reveal the presence of systematics behind the non-Gaussianity associated with the Cold Spot. Besides these test, it is important to remark that the angular size associated with this feature is $\approx 10^{\circ}$ in the sky. It is not trivial to think in a systematic effect affecting at this scale, and providing such localized feature in the sky as the Cold Spot.

Finally, the subsequent releases of the WMAP data (where the modelling of the instrumental properties have been improving with time) have shown that there are not changes in the non-Gaussianity deviation, except for a slight increasing on its significance, which reflects the higher signal-to-noise ratio that WMAP data is getting as observational time increases.

5.2. Foregrounds. Astrophysical contaminants or foregrounds are the next possible origin for the non-Gaussianity associated with the Cold Spot. It is well known that foregrounds are highly non-Gaussian signals. It is worth commenting that although the Cold Spot is negative, it is still possible to think in an additive source (as foregrounds are) as a feasible explanation. To understand this point, it is important to recall that the QVW map (that, as the author said before, is commonly adopted in the literature for cosmological analyses) is obtained as a noise-weighted linear combination of cleaned CMB maps at different frequencies. These maps (at 41,61 , and $94 \mathrm{GHz}$ ) are produced, as mentioned previously, via a template fitting (see, e.g., [75]). Therefore, any oversubtraction of foregrounds templates could cause a foreground residual in the form of a cold emission. (In some works, a VW map is adopted, that is, a map built as a noise-weighted linear combination of the cleaned V-and W-bands.)

Since, as mentioned before, the size of the Cold Spot is of several degrees, it is really hard to believe that this feature could be associated with residuals from point sources (i.e., from radio and infrared galaxies). Therefore, only galactic emissions (as synchrotron, free-free, and thermal 


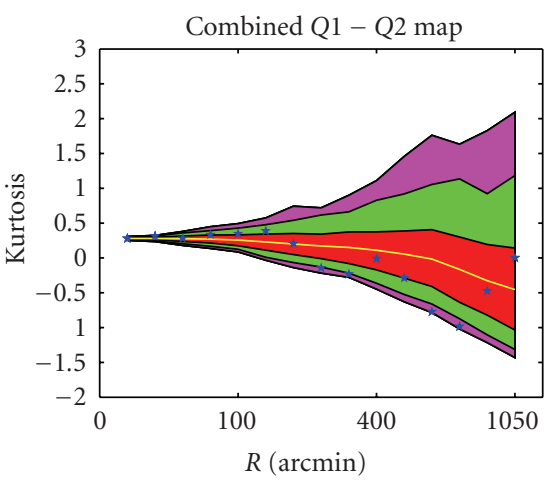

(a)

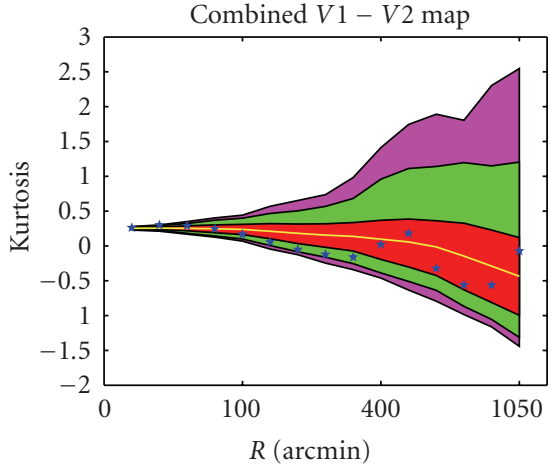

(b)

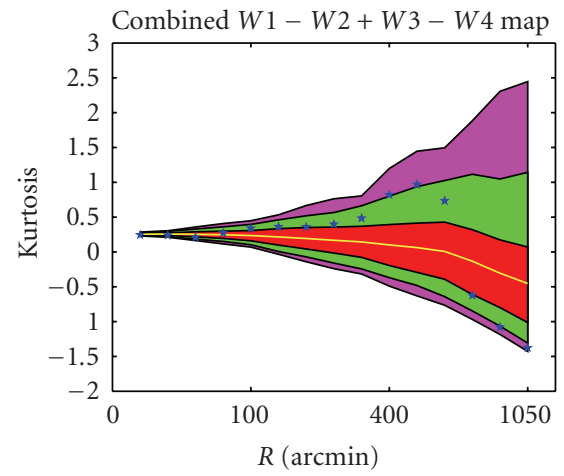

(c)

FIGURE 9: Null tests performed on difference maps, free of any foreground and cosmological signal. (a) represents the kurtosis of the SMHW coefficients, as a function of the scale $R$, for the $Q 1-Q 2$ map, that is, for a map built as the difference of the difference assemblies observations at $41 \mathrm{GHz}$. (b) is as the previous one, but for the $V 1-V 2$ difference map, that is, at $61 \mathrm{GHz}$. Finally, (c) provides the variation of the SMHW coefficients as a function of the wavelet scale, but for the difference map obtained with the 4 difference assemblies of WMAP at $90 \mathrm{GHz}$, that is, $W 1-W 2+W 3-W 4$. The blue asterisks represent values for the data whereas red, green and magenta regions represented the acceptance intervals at $32 \%, 5 \%$, and $1 \%$, respectively, determined by simulations.

and spinning dust) could be responsible for a large feature as the Cold Spot. However, notice that the Cold Spot is located at $57^{\circ}$ from the Galactic plane, and at a longitude of $209^{\circ}$. In other words, the Cold Spot is placed in a region of low Galactic contamination. According to the previous reasoning, it is already hard to make compatible the presence of the Cold Spot with a given Galactic emission. Even though, of course, the issue has been a matter of discussion. The author reviews here some of these analyses.

The most obvious test is, of course, to check whether there is or not any frequency dependence of the statistical estimators that indicated the Gaussian deviation. For instance, in $[28,82]$, the kurtosis of the SMHW coefficients $\left(K_{R}\right)$ was studied for the different $\mathrm{CMB}$-cleaned maps obtained at the Q-, V-, and W-bands.

The results obtained for this study are given in Figure 8. The kurtosis $K_{R}$ is presented for the 41,61 , and $94 \mathrm{GHz}$ channels, and for the noise-weighted lineal combination (the QVW map). It is remarkable the high similarity of the curves. The pattern of the kurtosis, as a function of the SMHW scale, is the same for all the maps. The same is observed for its normalization. An equivalent test can be done for the area of the SMHW coefficients $\left(A_{R}^{v}\right)$, as proposed by $[46,82]$. Results, at $R=300 \mathrm{arcmin}$, are presented in Figure 10. Notice that the agreement of the area of the SMHW coefficients (above threshold $v=3,3.5,4 \sigma_{R}$ ) among different frequency bands $(\mathrm{Q}, \mathrm{V}$, and $\mathrm{W})$ and the combined QVW map (represented by the solid lines in the figure) is very high. This kind of tests shows that there is not any evident frequency dependence of the statistics associated with the non-Gaussian deviation and, therefore, that such anomaly is fully consistent with the expected behaviour for a CMB feature.

Additional tests supporting this idea have been proposed in the literature. First [28], the kurtosis of the SMHW coefficients was analyzed for a CMB-free map, constructed as the combination of the 4 difference assemblies at the W-band

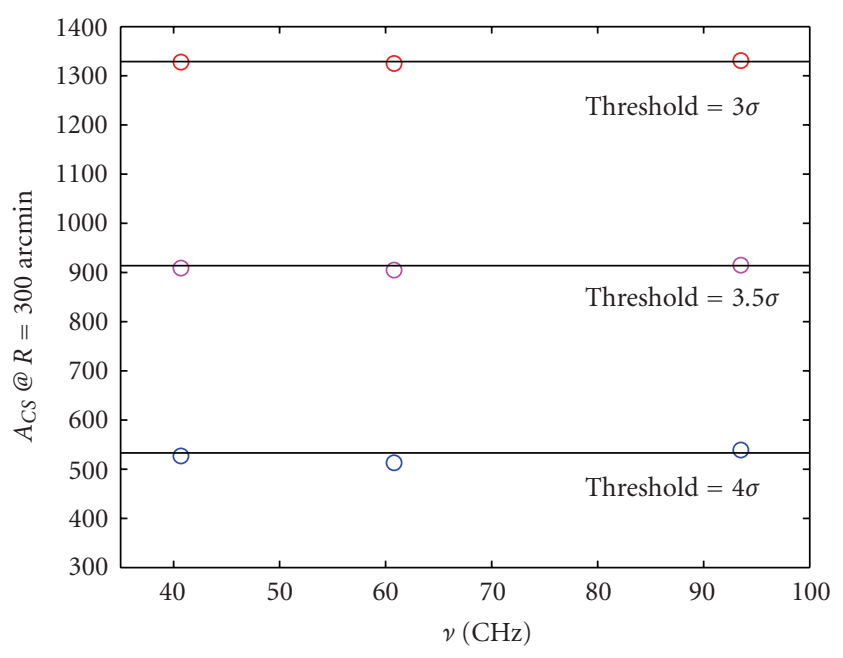

FIGURE 10: Frequency dependence of area of the SMHW coefficients. The plot shows the area of the wavelet coefficients at $R=$ 300 arcmin for different thresholds (from top to bottom: 3, 3.5, and $\left.4 \sigma_{R}\right)$, as a function of the frequency. The circles represent the values obtained for cleaned CMB maps obtained from the WMAP data at three cosmological frequencies (Q-, V-, and W-bands). The solid lines represent the areas, at different thresholds, for the noiseweighted QVW map.

minus the sum of the 4 ones at Q- and V-band (i.e., $W 1+$ $W 2+W 3+W 4-Q 1-Q 2-V 1-V 2)$. This kind of map could have a contribution of the Galactic contaminants (outside a given observing mask), since foreground emissions are not frequency independent. This analysis did not show any significant deviation from the expected behaviour from Gaussian simulations, and, therefore, discarding the presence of significant foreground residuals.

Second, as suggested by [82], different CMB recoveries from the WMAP data (where independent and alternative 
component separation approaches were followed) could be analyzed. In particular, the cleaned CMB maps obtained by [89] were probed. The kurtosis, the area, and the MAX of the SMHW coefficients do not change significantly from the different CMB maps.

Reference [82] explored a more complicate scenario: a situation in which combinations of different foreground emissions could mimic, in the region of the Cold Spot, the behaviour associated with the $\mathrm{CMB}$, that is, a frequency independent global emission. To check this possibility, several templates were used as tracers of the Galactic foregrounds, namely, the Rodhes/HartRa0 $2326 \mathrm{MHz}$ [90] radio survey for synchrotron, the $H_{\alpha}$ by [91] for the free-free, and the thermal dust model by [92]. Authors studied the expected contribution of foregrounds in the region of the Cold Spot, and they found that taking into account the uncertainties in the extrapolation of these templates from their original observations to the WMAP frequency range, it was possible to find a global Galactic emission that was nearly frequencyindependent from 41 to $94 \mathrm{GHz}$ (i.e., from Q- to Wbands). However, it was found that the emission was at a level of one order of magnitude below the Cold Spot temperature. It was checked that even accounting twice for that hypothetical foreground emission, it was not possible to reconcile observations with the Gaussian model.

All these tests on the impact of the foregrounds indicated that the non-Gaussian signal associated with the Cold Spot was fully consistent with a CMB like frequency dependence, and that the role played by astrophysical contaminants was negligible.

5.3. The Sunyaev-Zeldovich Effect. After checking that the possible impact of systematics and foregrounds on the nonGaussian detection is very low, the next step is to study whether secondary anisotropies of the CMB could explain the anomalous nature of the Cold Spot.

The Sunyaev-Zeldovich effect (SZ) could be a potential candidate to explain the anomaly (it is produced by the inverse Compton interaction of the CMB photons, as they cross the hot electron gas that is found in clusters of galaxies). Two major reasons support this possibility: first, the size of the Cold Spot is nearly compatible with the fluctuations caused by the nearest clusters of galaxies, and second, this fluctuations (in the frequency range covered by WMAP) produce cold spots [93].

There is no evidence for the existence of any large cluster in the direction of the Cold Spot. However, [94] reported the presence of a large concentration of galaxies in that direction-referred to as the Eridanus super-group-that could account for a total mass of $\approx 10^{14} M_{\odot}$ (see [95] for details).

This scenario implies, therefore, that the SZ could explain the nature of the Cold Spot, at least, partially. In other words, it could be possible that a combination of a SZ contribution plus a large (but not anomalous) CMB fluctuation could account for the Cold Spot emission. This was studied by [82], and the results are summarized in Figure 11. In this figure, the values of the SMHW coefficients $w(\theta, \phi ; R)$, at

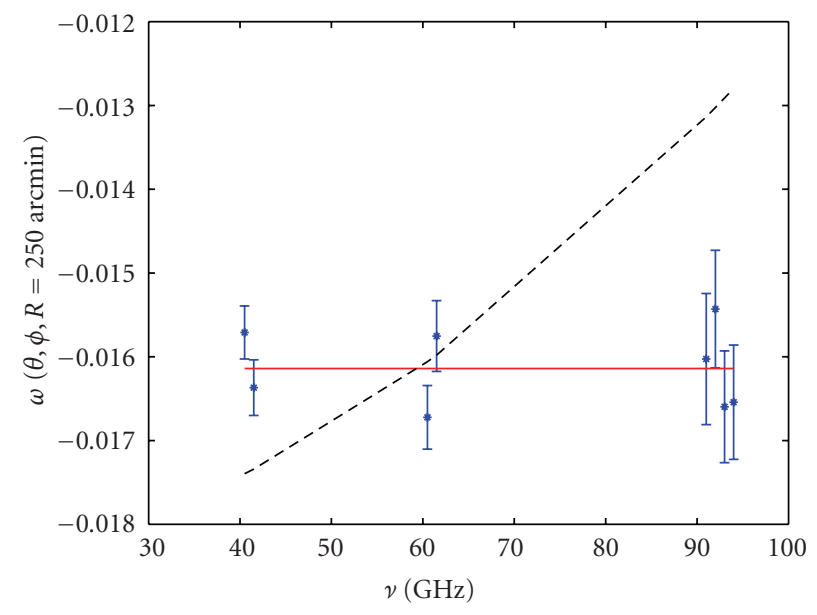

FIgURE 11: Best-fit of the WMAP data-across the 8 difference assemblies for the Q-, V-, and W-bands-to a CMB (solid line) and a SZ spectra (dashed line). The fit is performed to the minimum amplitude of the Cold Spot in wavelet space, at a scale of $R=$ 300 arcmin. Error bars are obtained from Gaussian simulations.

the position of the Cold Spot and at $R=300 \mathrm{arcmin}$, are given for the eight CMB cleaned maps at the Q-, V-, and $\mathrm{W}$-bands. The corresponding error bars were computed from $\mathrm{CMB}$ plus noise simulations, corresponding to the instrumental properties of these detectors, and convolved with the appropriate SMHW kernel.

Three different fits to the data were explored. First, a pure CMB spectrum was used, giving a very good fit with a reduced $\chi^{2}=1.00$. Such a fit is represented by the solid line in Figure 11. Second, a pure SZ spectrum was tested, obtaining a very poor fit (dashed line) with a reduced $\chi^{2}=9.12$. Finally, a joint fit to a CMB plus SZ spectra was explored, obtaining an amplitude for the $S Z$ spectrum consistent with zero and a reduced $\chi^{2}$ quite similar to the first case. These results confirm that the frequency dependence of the Cold Spot is consistent with a CMB-like spectrum. They also rule out the possibility that the $\mathrm{SZ}$ is playing any significant role.

5.4. The Late Evolution of the Large-Scale Structure. Another secondary anisotropy that could explain the anomalous nature of the Cold Spot is the one due to the nonlinear evolution of the gravitational field: the so-called Rees-Sciama effect (RS). In particular, it is known $[96,97]$ that voids in the large-scale structure could induce a negative nonlinear anisotropy in the CMB photons. The size of such secondary anisotropies depends on the proper size of the void and its redshift. Therefore, as for the SZ, the RS is another potential candidate to explain the anomalous nature of the Cold Spot.

Extra support for this hypothesis came from two different paths. On the one hand, theoretical works as [98, 99] proposed that a very large void (of $\approx 300 h^{-1} \mathrm{Mpc}$ ) and located at low redshift $(z \ll 1)$ could produce large negative CMB fluctuations such as the Cold Spot, even with modest density contrast values (i.e., in a quasilinear regime). On the other hand, it was suggested [100] that the NVSS catalogue 


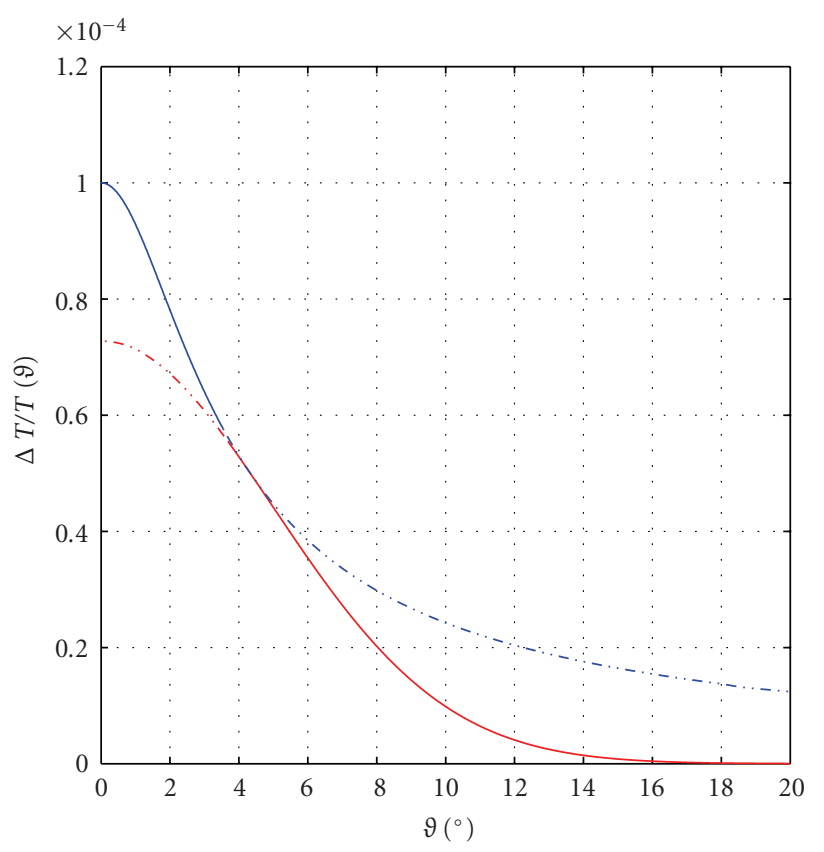

Figure 12: Radial profile (solid blue/red lines) of a cosmic texture, as given in (14). The parameters defining this profile are $\varepsilon=10^{-4}$ and $\vartheta_{c}=5^{\circ}$. The dashed lines represent the truncated values of the profiles given by (13) (blue) and the Gaussian function (red). See text for details.

[101] seems to show, at the position of the Cold Spot, a lack in the number count of radio galaxies.

Against these ideas, some criticisms can be made. First, this kind of voids are not observed and, even more, according to current $N$-body simulations [102] they are extremely rare events $(\approx 13 \sigma$, i.e., much more rare than the Cold Spot deviation itself, that was a $98.15 \%$ event!) in the standard cosmological framework. Second, the claim made on the NVSS data has been recently questioned by [103], suggesting that such finding was an artefact caused by possible systematics related with the NVSS data processing, the statistical procedure and the a posteriori selection of the Cold Spot position.

In addition, observational campaigns on the region of the Cold Spot were made by [104] with the MegaCam on the Canada-France-Hawaii Telescope and by [105] with the VIMOS spectrograph on the very large telescope. Both works have reported that the large-scale structure in that direction, and up to $z \approx 1$, is fully consistent with the standard model and that there is no evidence of such large voids as those required by the nonlinear evolution of the gravitational potential hypothesis.

\section{A Plausible Explanation: Cosmic Textures}

In the previous section, the author has presented an overview of the several works carried out to establish whether the non-Gaussianity detection associated with the Cold Spot could be explained in terms of systematics, foregrounds, and secondary anisotropies as the Sunyaev-Zeldovich and the Rees-Sciama effects. None of these possibilities seem to provide a satisfactory explanation, and, therefore, other sources should be investigated.

In this section, the author pays attention to the suggestion made by [106]: the Cold Spot could be caused by a cosmic texture. Cosmic textures [107] are a type of cosmic defects. They are supposed to be generated at some stage of the early Universe, associated with the symmetry-braking phase transitions that are predicted by certain theoretical models of high energy physics (see, e.g., [108] and references therein for a much more detailed explanation). In short, defects can be understood as space regions of a given phase state, surrounded by a space already in a new phase. In some cases, as for textures, these regions could collapse and, therefore, left an imprint on the CMB photons.

Among the different types of cosmic defects, textures are the most plausible candidate to explain the anomalous nature of the Cold Spot, since the interaction of the $\mathrm{CMB}$ photons with the time variation of the gravitational potential, associated with an eventual collapse of the texture, produces spots in the CMB fluctuations [109]. Even more, cosmic textures are expected to be a possible source of kurtosis deviation whereas the expected level of skewness is almost negligible (at least for values of the symmetrybreaking energy scale compatible with current observations). This is caused by the even probability of textures producing cold and hot spots and, therefore, providing a nearly symmetric distribution of temperature fluctuations. In fact, the equilateral $f_{\mathrm{NL}}$ expected from textures goes as $f_{\mathrm{NL}} \approx$ $1.5 \times 10^{-100} \psi_{0}^{6}[110]$, where $\psi_{0}$ is the symmetry-breaking energy scale, measured in Gev. For typical limits in $\psi_{0}$ imposed by the $\mathrm{CMB}$ angular power spectrum analysis (e.g., $[111,112])$, the expected equilateral $f_{\mathrm{NL}}$ is $\approx 10^{-9}$, that is, a tiny value well below the current constraints [113].

The isotropic shape of the temperature fluctuations related to these spots can be approximated, at least at small angular distances $\vartheta$, as [114]

$$
\frac{\Delta T}{T}(\vartheta)= \pm \varepsilon \frac{1}{\sqrt{1+4\left(\vartheta / \vartheta_{c}\right)^{2}}},
$$

where $\vartheta$ represents the angular distance from the centre of the spot, and $\vartheta_{c}$ is a characteristic scale parameter of the spotthat is related to the redshift of the spot and the dynamics of the Universe [106]. The amplitude $\varepsilon$ is proportional to the symmetry-breaking energy scale $\psi_{0}: \varepsilon=8 \pi^{2} G \psi_{0}^{2}$. It is worth remarking that, according to cosmic texture models, the amplitude $\varepsilon$ is the same for every single spot generated by the collapsing defects.

As mentioned above, the profile proposed in (13) is only valid up to small relative distances (e.g., $\vartheta \approx \vartheta_{c}$ ) (the author knowledge, there is not any analytical or numerical solution for the full profile of a given cosmic texture yet). In order to have a profile valid at larger angular distances, [106] proposed to extend the profile given by (13) from its halfmaximum, following a Gaussian function. The extension is done by imposing continuity, both, of the profile itself and of its first derivative. 
Taking into account these conditions, the assumed profile for the CMB temperature fluctuation caused by a collapsing cosmic texture would be given by

$$
\frac{\Delta T}{T}= \pm \begin{cases}\frac{\varepsilon}{\sqrt{1+4\left(\vartheta / \vartheta_{c}\right)^{2}},} & \text { if } \vartheta \leq \vartheta_{*} \\ \frac{\varepsilon}{2} e^{-\left(1 / 2 \vartheta_{c}^{2}\right)\left(\vartheta^{2}+\vartheta_{*}^{2}\right)}, & \text { if } \vartheta>\vartheta_{*},\end{cases}
$$

where $\vartheta_{*}=\sqrt{3} / 2 \vartheta_{c}$.

In Figure 12, the author shows the radial section of the above profile (solid line). The red and blue parts of the solid line correspond to the Gaussian function and to the profile of (13), respectively. The dashed lines represent the truncated regions for both curves. The cosmic texture parameters used in this profile are $\vartheta=5^{\circ}$ (i.e., similar to the SMHW scale at which the Cold Spot appears as anomalous) and $\varepsilon=10^{-4}$. This value corresponds to a symmetry-breaking energy scale of $\psi_{0}=1.13 \times 10^{16} \mathrm{GeV}$ that corresponds to a conservative upper limit imposed by $\mathrm{CMB}$ measurements (e.g., $[111,115])$.

The author remarks that the results obtained with a profile as the one given in (14), and that are reviewed in the next section, do not depend very much with the specific function adopted for the extrapolation. Similar results are obtained, for instance, when an exponential function or a SMHW-like kernel are used.

6.1. The Bayesian Framework. Attending to the issues discussed in the previous subsection, a cosmic texture could be a strong candidate to explain the Cold Spot: textures produce spots on the CMB temperature fluctuations, they are nonGaussian signals, and, depending on their amplitude (or the symmetry-breaking scale), they could be compatible with current constraints on the role played by cosmic defects on the structure formation and evolution of the Universe.

Reference [106] proposed to make use of the texture profile of (14) to perform a hypothesis test to decide whether the WMAP data (in the position of the Cold Spot) is more likely to be described by a large (but not anomalous) $\mathrm{CMB}$ spot (i.e., the null $H_{0}$ hypothesis) or by a cosmic texture of amplitude $\varepsilon$ and size $\vartheta$ added to a random Gaussian and isotropic CMB field (i.e., the alternative $H_{1}$ hypothesis).

The optimal way of performing such hypotheses test is within the Bayesian framework. Bayes' theorem states that, given a data set $D$ and some unknown parameters $\Theta$ (defining a given model in the context of a given hypothesis $H_{i}$ ), the posterior probability of the parameters/model given the data $P\left(\Theta \mid D, H_{i}\right)$, is related to the likelihood $P\left(D \mid \Theta, H_{i}\right)$ (i.e., the probability of the data given the parameters/model) as

$$
P\left(\Theta \mid D, H_{i}\right)=\frac{P\left(D \mid \Theta, H_{i}\right) P\left(\Theta \mid H_{i}\right)}{P\left(D \mid H_{i}\right)},
$$

where $P\left(\Theta \mid H_{i}\right)$ is a measurement of our a priori knowledge about the parameters/model (i.e., the prior), and $P\left(D \mid H_{i}\right)$ is a constant (i.e., it does not depend on the parameters/model) called Bayesian evidence (BE). The BE is nothing but the average likelihood with respect to the prior

$$
P\left(D \mid H_{i}\right)=\int P\left(D \mid \Theta, H_{i}\right) P\left(\Theta \mid H_{i}\right) \mathrm{d} \Theta,
$$

and it is a largely used mechanism to perform hypotheses test. In particular, its role on different cosmology fields has been quite remarkable during the last years (e.g., [116] for dark energy studies, [117] for anisotropic models of the Universe expansion, [118] for studying different reionization models, [119] for point source detection, and [120] for exploring nonstandard inflationary models).

The importance of BE for hypotheses test is clear. First, it is obvious that the quantity that we would like to obtain is a measurement of the probability of a given hypothesis $H_{0}$, given the data, that is, $P\left(H_{0} \mid D\right)$. This probability can be written, attending to the probability multiplication rule, as

$$
P\left(H_{0} \mid D\right)=P\left(D \mid H_{0}\right) \frac{P\left(H_{0}\right)}{P(D)},
$$

that is, it is proportional to the $\mathrm{BE}$ and to the probability of the hypothesis and inversely proportional to the probability of the data. Under certain circumstances, the probability of the hypothesis could be known, but, however, it is not the case for the probability of the data. In other words, we only can learn about the probability of the hypothesis $H_{0}$ up to a factor. Therefore, what we can extract is a relative measurement of the probability of two hypotheses $\left(H_{0}\right.$ and $H_{1}$ ), given the same data set $D$. This relative measurement is called the posterior probability ratio, $\rho$, and reads

$$
\rho \equiv \frac{P\left(H_{1} \mid D\right)}{P\left(H_{0} \mid D\right)}=\frac{E_{1}}{E_{0}} \frac{P\left(H_{1}\right)}{P\left(H_{0}\right)}
$$

where, for simplicity, the author rewrite the $\mathrm{BE}, P\left(D \mid H_{i}\right)$, as $E_{i}$. Hence, if $\rho>1$, we can conclude that the hypothesis $H_{1}$ is favoured by the data with respect to $H_{0}$. In some cases, there is not a clear choice for the probability of the hypotheses. In this case, empirical rules for the ratio of evidences-as the Jeffreys' scale [121] — are usually adopted.

Therefore, the procedure required to explore whether the Cold Spot is more likely to be explained in terms of a cosmic texture $\left(H_{1}\right)$ rather than by a Gaussian CMB fluctuation $\left(H_{0}\right)$ is clear: first, the likelihood is computed for both hypotheses; second, the $\mathrm{BE}$ is estimated, taking into account the adequate priors (16); finally, the posterior probability ratio (18) is evaluated, making use of suitable a priori probabilities for $H_{0}$ and $H_{1}$.

This was the procedure followed in [106]. It is straightforward to show that since the noise term is caused by standard CMB Gaussian fluctuations and instrumental Gaussian 
noise, the likelihood function reads as

$$
P\left(D \mid \Theta, H_{i}\right) \propto \exp \left(-\frac{\chi^{2}}{2}\right),
$$

where $\chi^{2}=(D-T(\Theta)) C^{-1}(D-T(\Theta))^{\mathrm{T}}$. The correlation matrix $C$ accounts for the full Gaussian $\mathrm{CMB}$ and noise correlations-that is, $C=S+N$, where $s_{i j} \propto \sum C_{\ell} P_{\ell}\left(\cos \theta_{i j}\right)$ and $n_{i j}=\sigma_{i}^{2} \delta_{i j}$, being $P_{\ell}$ the Legendre polynomials, $\theta_{i j}$ the angular distance between the pixels $i$ and $j, \sigma_{i}^{2}$ the instrumental noise contribution to pixel $i$, and $\delta_{i j}$ represents the Kronecker delta. $D$ represents the data (i.e., the QVW map), and the function $T(\Theta)$ represents the model behind the hypotheses_-that is, (14) for $H_{1}$, and $\equiv 0$ for $H_{0}$. Finally, $\mathrm{T}$ denotes standard matrix transpose.

The priors adopted by [106] for the parameters $\Theta \equiv$ $\left(\varepsilon, \vartheta_{c}\right)$ were chosen attending to observational constraints and cosmic texture simulations. In particular, the prior on the amplitude was $|\mathcal{E}| \leq 10^{-4}$ whereas the prior of the size $1^{\circ} \leq \vartheta_{c} \leq 15^{\circ}$ was assumed. The amplitude prior was uniformly distributed, and, as mentioned above, it is a conservative constraint imposed from the contribution of cosmic defects to the CMB angular power spectrum. The size $\vartheta_{c}$ follows a scale-invariant law, and the limits come from texture simulations. Textures below $1^{\circ}$ should be smeared out by photon diffusion, and, in addition, they would be related to collapsing events above redshift $\approx 1000$, which would not affect the CMB image. The upper limit is due to the unlikely probability of generating such large textures in the finite celestial sphere. Even so, [106] tested that results were not specially sensitivity to the prior selection, since the likelihood was clearly peaked, within a region of the parameter space clearly allowed by observations and texture models.

The marginalization of the posterior probability in (15), led to the determination of the texture parameters, obtaining $\varepsilon=7.3_{-3.6}^{+2.5} \times 10^{-5}$ and $\vartheta_{c}=4.9^{\circ}{ }_{-2.4^{\circ}}$ at $95 \%$ confidence. The BE ratio was 150 , which, in terms of the empirical rules [121], is a strong indication that the texture hypothesis for the Cold Spot is favoured over the isotropic and Gaussian CMB fluctuation option. Adopting a ratio for the probability of the hypotheses given by the fraction of the sky that is covered by a cosmic texture as large as the one required for the Cold Spot $(\approx 0.017)$, the posterior probability ratio was $\rho=2.5$, which also favours the texture hypothesis.

It is worth mentioning that the estimated value for the texture amplitude could be affected by selection bias. In [106], it is established that such bias could provide an overestimate of the texture amplitude by a factor of 2 . This bias is caused because the texture amplitude is estimated in a low signal-to-noise regime, where the features placed in large background fluctuations are more easily detected. Even so, the estimated value for the texture amplitude, $\varepsilon=7.3 \times 10^{-5}$, would imply a symmetry-breaking energy scale of $\psi_{0}=$ $8.7 \times 10^{15} \mathrm{GeV}$, which, on the one hand, is fully compatible with more recent constraints imposed from the analysis of the CMB temperature and polarization power spectra (e.g., [112]), and, on the other hand, is in agreement with the predictions of most of the models for particle physics. In addition, by relating the angular size of the $\mathrm{CMB}$ texture profile to the cosmological parameters defining the geometry and evolution of the Universe, it was possible to establish that the texture collapse (that generated the CMB profile in (14)) occurred at redshift $z \approx 6$.

Figure 13 shows the effect of correcting the QVW map from the texture emission. In the left panel, the region of the sky where the Cold Spot was identified is shown. The middle panel presents the best fit of the texture profile, according to the parameters previously mentioned. On the right panel, the author presents the resulting map after the subtraction of the estimated cosmic texture contribution. The Cold Spot is noticeably reduced. More quantitative measurements were made by [106]. It was proved that if cosmic textures (adequate to the parameters fixed by the Cold Spot analysis) were added to isotropic and Gaussian simulations of CMB signals, as seen in the QVW WMAP map, then the kurtosis of the SMHW became compatible. Even more, [122] showed that the WMAP map (corrected from the cosmic texture contribution in the location of the Cold Spot) was not compatible with anisotropic patterns for nonstandard expansions of the Universe (in particular, for Bianchi $\mathrm{VII}_{h}$ models), as it was previously the case for the uncorrected data (e.g., $[117,123]$ ).

Finally, the author remarks that [95] proposed a similar approach as the one described in this section to study (attending to spatial templates) whether SZ and RS could provided more suitable hypotheses than the standard isotropic and Gaussian model. This Bayesian analysis indicated that neither of these hypotheses is favoured.

6.2. Followup Tests. The studies described in Section 5 and in the previous subsection, clearly indicates that among all the realistic sources that could explain the anomalous nature of the Cold Spot, only the cosmic texture hypothesis remains as a feasible option. The results obtained by [106], and reviewed in the previous subsection, have to be understood as a (clear) indication that the cosmic texture is plausible. However, before accepting it as the final explanation, it should be confirmed by additional tests. In particular, if the cosmic texture hypothesis would be the right one, then there are some clear predictions that could be tested (at least in the near feature, once ongoing/upcoming experiments as SPT, ACT, QUIJOTE, and ALMA are fully operative).

In this subsection, the author comments on the three most obvious followup tests that could help to discard, or accept, the cosmic texture. These follow-up tests are: the searching of more textures, the local polarization of the $\mathrm{CMB}$, and the local CMB lensing. These foreseen effects were firstly pointed out by [106].

6.2.1. Looking for More Textures. If a texture were found in the location of the Cold Spot, then, attending to cosmic texture models, there should be more cold and hot spots randomly distributed across the sky. In fact, the distribution of $\mathrm{CMB}$ spots caused by cosmic textures follows a scaleinvariant law

$$
N_{\mathrm{sp}}\left(>\vartheta_{c}\right)=\frac{4 \pi \nu \kappa^{3}}{3 \vartheta_{c}^{2}}
$$




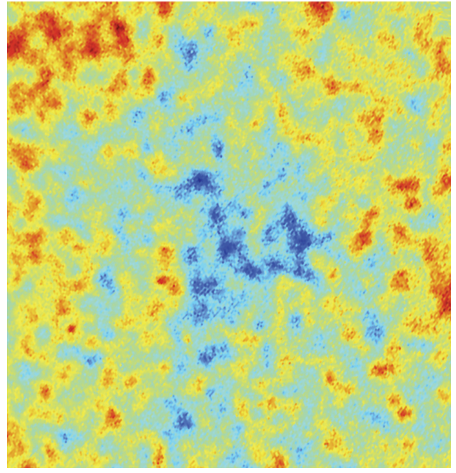

(a)

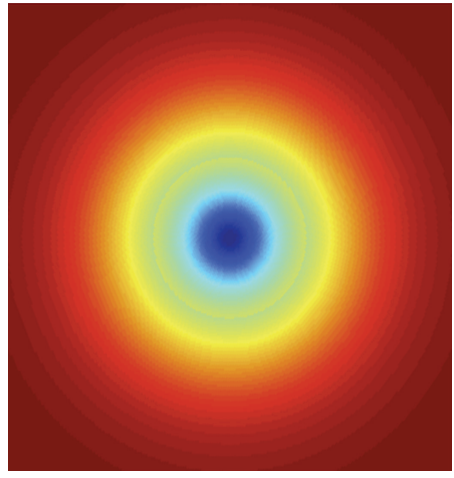

(b)

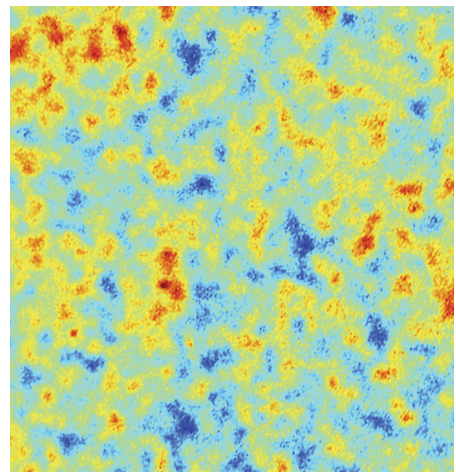

(c)

FIGURE 13: (a) CMB temperature fluctuations obtained from WMAP data, centred in the position of the Cold Spot. (b) best fit of a texture profile to the data, according to (14). (c) CMB map, at the position of the Cold Spot, after the subtraction of the best-fit texture model.

that is, the number of cold/hot spots with a scale equal or larger than $\vartheta_{c}$, is inversely proportional to $\vartheta_{c}^{2}$ (see [124] for the grounds and [106] for a derivation). The $v$ and $\kappa$ parameters are associated with the physics of the cosmic texture models, and their specific meaning is out of the scope of this paper. The author remarks here that according to state-of-the-art simulations, these parameters are well consistent with $\nu \approx 2$ and $\kappa \approx 0.1$ values, when $\vartheta_{c}$ is expressed in radians. Before continuing the discussion, the author stresses that the number of expected cold/hot spots with a scale $\vartheta_{c}$ equal or larger than $5^{\circ}$ (i.e., as the Cold Spot scale) is $\approx 1$. In other words, the fact that we only found a Cold Spot of a scale similar or larger than the Cold Spot size is fully consistent with the cosmic texture scenario. This fact, of course, is an extra support for the texture hypothesis causing the Cold Spot emission.

Let us come back to (20). A straightforward calculation tells as that if the cosmic texture hypothesis is correct, then the $\mathrm{CMB}$ temperature fluctuations should contain $\approx 28$ spots with a scale $\vartheta_{c} \geq 1^{\circ}$, and $\approx 7$ spots with a scale $\vartheta_{c} \geq 2^{\circ}$. Poisson errors can be safely assumed for these numbers. Therefore, the number of cold/hot spots expected in the WMAP data are $1 \lesssim N_{\text {sp }}\left(>2^{\circ}\right) \lesssim 13$ at the $95 \%$ confidence level. Current work is in progress to check this prediction, by using a fast cluster nesting sampling algorithm-MULTINEST [125]—to explore the posterior probability ratio. There are well-founded hopes to find new textures. In particular, some non-Gaussian analyses as [27, 48,88 ] reported some hot/cold spots (in addition to the Cold Spot) as potentially anomalous.

6.2.2. The Polarization of the $C M B$. The effect of a collapsing texture on the passing by $\mathrm{CMB}$ photons is nothing but a secondary anisotropy of the CMB fluctuations, whose origin is merely gravitational. Hence, the effect of such gravitational phenomenon on the E-mode polarization is almost negligible (only vector modes would be affected, which are well below the scalar mode contribution).

Strictly speaking, this lack of polarization is not a unique signature produced by cosmic textures. As the author said, any secondary anisotropy of gravitational origin would cause it. However, these other effects (as huge voids) are quite implausible explanations (see Section 5.4). For that reason, this effect is a valid follow-up test to probe the texture hypothesis.

The procedure is simple: to compare the E-mode polarization in the position of a temperature spot (as large and extreme as the Cold Spot is), under two different hypotheses, the null or $H_{0}$ one (i.e., the temperature spot is caused by a Gaussian fluctuation) and the alternative or $H_{1}$ option (i.e., the temperature spot is a secondary anisotropy caused by the collapse of an evolving texture). In fact, as proposed by [126], the best discriminating measurement is the TE correlation, rather than simply the E signal. This crosscorrelation is expected to be close to zero for the $H_{1}$ hypothesis. The approach suggested in this work was to estimate the correlation of the $\mathrm{T}$ and $\mathrm{E}$ profiles around the position of the spot temperature signal. This statistic was computed for many simulations according to the $H_{0}$ and $H_{1}$ hypotheses, and a hypothesis test was performed, via the definition of an optimal Fisher discriminant statistic (e.g., [127]).

The method was applied to probe the capabilities of current and upcoming $\mathrm{CMB}$ experiments for discriminating between the two hypotheses. In particular, the cases of WMAP, Planck [128], and QUIJOTE [129] were considered.

As the major conclusion, it can be established that the discrimination power of the T-E correlation is not very high. In fact, for an ideal noise-free experiment, at a power of the test of 0.5 , the significance level is (up most) $0.8 \%$. The reason for this limitation is that the characteristic size of the Cold Spot is $\approx 10^{\circ}$, which, roughly, corresponds to multipoles of $\ell \approx 40$. It happens that at this multipole scales, the T-E angular cross-power spectrum is very close to zero, already for $H_{0}$ and, therefore, it is hard to discriminate it from $H_{1}$.

The results are graphically summarized in Figure 14. The author represent the significance level or $p$-value (for a power of the test of 0.5) as a function of the instrumental noise sensitivity for the E-mode polarization. The three experiments previously mentioned are indicated as vertical lines. Notice that the WMAP instrumental characteristics 


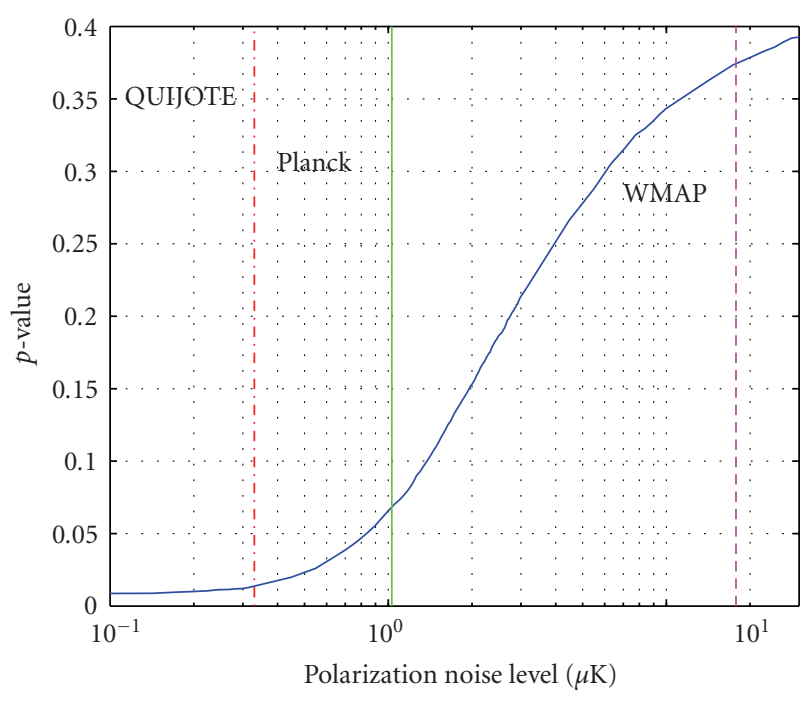

FIgURE 14: The curve indicates the probability (the $p$-value) for rejecting the texture hypothesis, attending to the T-E correlation, as a function of the instrumental noise sensitivity in polarization. From left to right, the vertical lines indicate the noise level associated with QUIJOTE, Planck, and WMAP experiments.

do not allow for any significant discrimination between hypotheses. With Planck, it would be possible to reach a modest $7 \%$ significance level. Finally, the upcoming QUIJOTE experiment would allow for a $1.4 \%$ detection. However, if the significance of the Cold Spot obtained via the first non-Gaussianity wavelet analysis (i.e., $1.85 \%$, as mentioned in Section 4) is also considered, [126] claims that a joint $\mathrm{T}$ and $\mathrm{T}$-E significance detections of $0.025 \%$ and $0.12 \%$ could be imposed on the texture hypothesis, for the QUIJOTE and Planck experiments, respectively.

6.2.3. Gravitational Lensing. Besides the lack of polarization discussed in the previous subsection, the lensing of the $\mathrm{CMB}$ photons would be another foreseen effect caused by the gravitational field generated by the collapsing texture. This point was recently addressed by [130]. This work studied the capabilities of small-scale CMB experiments as ACT to detect a possible lensing effect occurring at the position of the Cold Spot, caused by the gravitational field of a texture placed at $z=6$, and with a typical scale of $5^{\circ}$, associated with a symmetry-breaking energy scale of $\psi_{0}=4.5 \times 10^{15} \mathrm{GeV}$ (i.e., comparable to the parameters determined by [106]).

As for the polarization test, the power of the lensing analysis to probe the existence of the texture is relatively modest. For instance, detection is made at the $3 \sigma$ level after 1000 minutes of integration time. In other words, this test would require of a dedicated observational campaign.

\section{Conclusions}

In this paper the author has presented a comprehensive overview of the Cold Spot. Since its detection in 2003 by
[28], this feature proved to be one of the most intriguing anomalies found in the WMAP data.

The Cold Spot was detected after performing a nonGaussianity test on a cleaned CMB (obtained via a template fitting of the WMAP difference assemblies at Q-, V-, and $\mathrm{W}$-bands). The non-Gaussian analysis was performed by comparing the values obtained for the skewness and the kurtosis of the SMHW coefficients (at several scales) for such cleaned $\mathrm{CMB}$ map to the distribution expected from isotropic and Gaussian $\mathrm{CMB}$ realizations. This analysis indicated an excess of the kurtosis at SMHW scales $R$ of around $300 \mathrm{arcmin}$. Subsequent analysis of the SMHW coefficients, based on the area above/below a given threshold, the Higher Criticism, and the maximum value, agreed in detecting the non-Gaussian deviation occurring at the same scales, and confirmed the peculiar role played by the Cold Spot. Avoiding any possible a posteriori choice of statistics, a conservative significance detection level of $1.85 \%$ was placed by [76].

The Cold Spot was found to be highly isotropic [82], and the impact of possible systematics and residual foreground contamination was discarded $[28,46,76,82]$. Equivalently, some secondary anisotropies (potentially responsible for the Cold Spot emission) as the Sunyaev-Zeldovich and the ReesSciama effects, that, in principle, could be accepted as valid solutions, were show to be unlikely explanations $[82,95]$.

A plausible explanation in terms of a cosmic defect was addressed by [106]. It would imply the presence of a collapsing cosmic texture at redshift $z \approx 6$, with a typical scale of $\approx 5^{\circ}$, corresponding to a symmetry-breaking energy scale of $\psi_{0} \approx 9 \times 10^{15}$. In addition, some follow-up tests were proposed to confirm or discard the cosmic texture hypothesis. In particular, one should expect to have more spots generated by evolving textures in CMB observations (e.g., $\approx 7$ with scales $\vartheta \gtrsim 2^{\circ}$ ), a lack of E-mode polarization is predicted, as compared to the values associated with spots derived from a Gaussian CMB field; and, finally, a lensing of the $\mathrm{CMB}$ photons is potentially detectable with future small scale CMB experiments as ACT or SPT.

Summarizing, the study of the Cold Spot has provided a wealth of information, and the upcoming high-quality $\mathrm{CMB}$ data, as the one expected from Planck, guarantee that still more knowledge will come from the analysis of this very interesting feature.

\section{Acknowledgments}

The author thanks R. Belén Barreiro, Marcos Cruz, and Enrique Martínez-González for a careful reading of this paper. The issue reviewed in this paper is the result of a very fruitful and exciting collaboration with them (and with other colleagues) during the last 7 years, and the author presumes it is not over yet, since new data is expected to come in the near future. The author acknowledges partial financial support from the Spanish Ministerio de Ciencia e Innovación Project no. AYA2007-68058-C03-02. The author also thanks financial support from the Ramón y Cajal programme. 


\section{References}

[1] E. Komatsu, J. Dunkley, M. R. Nolta et al., "Five-year wilkinson microwave anisotropy probe observations: cosmological interpretation," Astrophysical Journal, Supplement Series, vol. 180, no. 2, pp. 330-376, 2009.

[2] D. Larson, J. Dunkley, G. Hinshaw et al., "Seven-year Wilkinson microwave anisotropy probe (WMAP) observations: power spectra and WMAP-derived parameters," http://arxiv.org/abs/1001.4635.

[3] H. K. Eriksen, F. K. Hansen, A. J. Banday, K. M. Górski, and P. B. Lilje, "Asymmetries in the cosmic microwave background anisotropy field," The Astrophysical Journal, vol. 605, no. 1, pp. 14-20, 2004.

[4] F. K. Hansen, P. Cabella, D. Marinucci, and N. Vittorio, "Asymmetries in the local curvature of the wilkinson microwave anisotropy probe data," The Astrophysical Journal, vol. 607, no. 2, pp. L67-L70, 2004.

[5] H. K. Eriksen, D. I. Nivikov, P. B. Lilje, A. J. Banday, and K. M. Górski, "Testing for non-Gaussianity in the Wilkinson microwave anisotropy probe data: Minkowski functionals and the length of the skeleton," The Astrophysical Journal, vol. 612, no. 1, pp. 64-80, 2004.

[6] F. K. Hansen, A. J. Banday, and K. M. Górski, “Testing the cosmological principle of isotropy: local power-spectrum estimates of the WMAP data," Monthly Notices of the Royal Astronomical Society, vol. 354, no. 3, pp. 641-665, 2004.

[7] E. P. Donoghue and J. F. Donoghue, "Isotropy of the early universe from CMB anisotropies," Physical Review D, vol. 71, no. 4, Article ID 043002, 2005.

[8] J. Hoftuft, H. K. Eriksen, A. J. Banday, K. M. Górski, F. K. Hansen, and P. B. Lilje, "Increasing evidence for hemispherical power asymmetry in the five-year wmap data," The Astrophysical Journal, vol. 699, no. 2, pp. 985-989, 2009.

[9] D. Pietrobon, P. Cabella, A. Balbi, R. Crittenden, G. de Gasperis, and N. Vittorio, "Needlet bispectrum asymmetries in the WMAP 5-year data," Monthly Notices of the Royal Astronomical Society, vol. 402, no. 1, pp. L34-L38, 2010.

[10] P. Vielva and J. L. Sanz, "Constraints on fNL and gNL from the analysis of the N-pdf of the CMB large-scale anisotropies," Monthly Notices of the Royal Astronomical Society, vol. 404, no. 2, pp. 895-907, 2010.

[11] F. Paci, A. Gruppuso, F. Finelli et al., "Power asymmetries in the cosmic microwave background temperature and polarization patterns," Monthly Notices of the Royal Astronomical Society, vol. 407, no. 1, pp. 399-404, 2010.

[12] P. Bielewicz, K. M. Górski, and A. J. Banday, "Low-order multipole maps of cosmic microwave background anisotropy derived from WMAP," Monthly Notices of the Royal Astronomical Society, vol. 355, no. 4, pp. 1283-1302, 2004.

[13] D. J. Schwarz, G. D. Starkman, D. Huterer, and C. J. Copi, "Is the low- $\ell$ microwave background cosmic?" Physical Review Letters, vol. 93, no. 22, Article ID 221301, 2004.

[14] C. J. Copi, D. Huterer, and G. D. Starkman, "Multipole vectors: a new representation of the CMB sky and evidence for statistical anisotropy or non-Gaussianity at $2 \leq \ell \leq 8$," Physical Review D, vol. 70, no. 4, Article ID 043515, 13 pages, 2004.

[15] A. de Oliveira-Costa, M. Tegmark, M. Zaldarriaga, and A. Hamilton, "Significance of the largest scale CMB fluctuations in WMAP," Physical Review D, vol. 69, no. 6, Article ID 063516, 2004.
[16] P. Bielewicz, H. K. Eriksen, A. J. Banday, K. M. Górski, and P. B. Lilje, "Multipole vector anomalies in the first-year WMAP data: a cut-sky analysis," The Astrophysical Journal, vol. 635, no. 2, pp. 750-760, 2005.

[17] K. Land and J. Magueijo, "Cubic anomalies in the Wilkinson microwave anisotropy probe," Monthly Notices of the Royal Astronomical Society, vol. 357, no. 3, pp. 994-1002, 2005.

[18] C. J. Copi, D. Huterer, D. J. Schwarz, and G. D. Starkman, "On the large-angle anomalies of the microwave sky," Monthly Notices of the Royal Astronomical Society, vol. 367, no. 1, pp. 79-102, 2006.

[19] L. R. Abramo, A. Bernui, I. S. Ferreira, T. Villela, and C. A. Wuensche, "Alignment tests for low CMB multipoles," Physical Review D, vol. 74, no. 6, Article ID 063506, 2006.

[20] A. Gruppuso and C. Burigana, "Large scale alignment anomalies of CMB anisotropies: a new test for residuals applied to WMAP 5yr maps," Journal of Cosmology and Astroparticle Physics, vol. 2009, no. 8, article 004, 2009.

[21] M. Frommert and T. A. Enßlin, "The axis of evil—a polarization perspective," Monthly Notices of the Royal Astronomical Society, vol. 403, no. 4, pp. 1739-1748, 2010.

[22] C. Monteserín, R. B. Barreiro, P. Vielva, E. MartínezGonzález, M. P. Hobson, and A. N. Lasenby, "A low cosmic microwave background variance in the Wilkinson microwave anisotropy probe data," Monthly Notices of the Royal Astronomical Society, vol. 387, no. 1, pp. 209-219, 2008.

[23] Y. Ayaita, M. Weber, and C. Wetterich, "Too few spots in the cosmic microwave background," Physical Review D, vol. 81, no. 2, Article ID 023507, 2010.

[24] L. Cayón, "Variograms of the cosmic microwave background temperature fluctuations: confirmation of deviations from statistical isotropy," Monthly Notices of the Royal Astronomical Society, vol. 405, no. 2, pp. 1084-1088, 2010.

[25] M. Cruz, P. Vielva, E. Martínez-González, and R. B. Barreiro, "Anomalous variance in the WMAP data caused by Galactic Foreground residuals," Monthly Notices of the Royal Astronomical Society, http://arxiv.org/abs/1005.1264. Submitted.

[26] Y. Wiaux, P. Vielva, E. Martínez-González, and P. Vandergheynst, "Global universe anisotropy probed by the alignment of structures in the cosmic microwave background," Physical Review Letters, vol. 96, no. 15, Article ID 151303, 2006.

[27] P. Vielva, Y. Wiaux, E. Martínez-González, and P. Vandergheynst, "Alignment and signed-intensity anomalies in Wilkinson microwave anisotropy probe data," Monthly Notices of the Royal Astronomical Society, vol. 381, no. 3, pp. 932-942, 2007.

[28] P. Vielva, E. Martínez-González, R. B. Barreiro, J. L. Sanz, and L. Cayón, "Detection of non-gaussianity in the Wilkinson microwave anisotropy probe first-year data using spherical wavelets," The Astrophysical Journal, vol. 609, no. 1, pp. 2234, 2004.

[29] A. Liddle and D. H. Lyth, Cosmological Inflation and LargeScale Structure, Cambridge University Press, Cambridge, UK, 2000.

[30] P. G. Ferreira, J. Magueijo, and J. Silk, "Cumulants as nonGaussian qualifiers," Physical Review D, vol. 56, no. 8, pp. 4592-4603, 1997.

[31] L. Popa, "Power fluctuations in the wavelet space: large-scale non-gaussian statistics," New Astronomy, vol. 3, no. 7, pp. 563-570, 1998.

[32] J. Pando, D. Valls-Gabaud, and L.-Z. Fang, "Evidence for scale-scale correlations in the cosmic microwave background 
radiation," Physical Review Letters, vol. 81, no. 21, pp. 45684571, 1998.

[33] C. L. Bennett, A. Banday, K. M. Gorski et al., "Four-year COBE DMR cosmic microwave background observations: maps and basic results," Astrophysical Journal Letters , vol. 464, pp. L1-L4, 1996.

[34] L. Tenorio, A. H. Jaffe, S. Hanany, and C. H. Lineweaver, "Applications of wavelets to the analysis of cosmic microwave background maps," Monthly Notices of the Royal Astronomical Society, vol. 310, no. 3, pp. 823-834, 1999.

[35] L. Cayón, J. L. Sanz, R. B. Barreiro et al., "Isotropic wavelets: a powerful tool to extract point sources from cosmic microwave background maps," Monthly Notices of the Royal Astronomical Society, vol. 315, no. 4, pp. 757-761, 2000.

[36] P. Vielva, E. Martínez-González, J. E. Gallegos, L. Toffolatti, and J. L. Sanz, "Point source detection using the Spherical Mexican Hat Wavelet on simulated all-sky Planck maps," Monthly Notices of the Royal Astronomical Society, vol. 344, no. 1, pp. 89-104, 2003.

[37] J. González-Nuevo, F. Argüeso, M. López-Caniego et al., "The Mexican hat wavelet family: application to point-source detection in cosmic microwave background maps," Monthly Notices of the Royal Astronomical Society, vol. 369, no. 4, pp. 1603-1610, 2006.

[38] M. López-Caniego, D. Herranz, J. González-Nuevo et al., "Comparison of filters for the detection of point sources in Planck simulations," Monthly Notices of the Royal Astronomical Society, vol. 370, no. 4, pp. 2047-2063, 2006.

[39] S. Pires, J. B. Juin, D. Yvon, Y. Moudden, S. Anthoine, and E. Pierpaoli, "Sunyaev-Zel'dovich cluster reconstruction in multiband bolometer camera surveys," Astronomy \& Astrophysics, vol. 455, no. 2, pp. 741-755, 2006.

[40] N. Aghanim and O. Forni, "Searching for the non-Gaussian signature of the CMB secondary anisotropies," Astronomy \& Astrophysics, vol. 347, no. 2, pp. 409-418, 1999.

[41] P. Mukherjee, M. P. Hobson, and A. N. Lasenby, "Do wavelets really detect non-Gaussianity in the 4-year COBE data?" Monthly Notices of the Royal Astronomical Society, vol. 318, no. 4, pp. 1157-1163, 2000.

[42] R. B. Barreiro, M. P. Hobson, A. N. Lasenby, A. J. Banday, K. M. Górski, and G. Hinshaw, "Testing the Gaussianity of the COBE DMR data with spherical wavelets," Monthly Notices of the Royal Astronomical Society, vol. 318, no. 2, pp. 475-480, 2000.

[43] R. B. Barreiro and M. P. Hobson, "The discriminating power of wavelets to detect non-Gaussianity in the cosmic microwave background," Monthly Notices of the Royal Astronomical Society, vol. 327, no. 3, pp. 813-828, 2001.

[44] L. Cayón, J. L. Sanz, E. Martínez-González et al., "Spherical Mexican hat wavelet: an application to detect nonGaussianity in the COBE-DMR maps," Monthly Notices of the Royal Astronomical Society, vol. 326, no. 4, pp. 1243-1248, 2001.

[45] E. Martínez-González, J. E. Gallegos, F. Argüeso, L. Cayón, and J. L. Sanz, "The performance of spherical wavelets to detect non-Gaussianity in the cosmic microwave background sky," Monthly Notices of the Royal Astronomical Society, vol. 336, no. 1, pp. 22-32, 2002.

[46] M. Cruz, E. Martínez-González, P. Vielva, and L. Cayón, "Detection of a non-Gaussian spot in WMAP," Monthly Notices of the Royal Astronomical Society, vol. 356, no. 1, pp. 29-40, 2005.

[47] J. D. McEwen, M. P. Hobson, A. N. Lasenby, and D. J. Mortlock, "A high-significance detection of non-Gaussianity in the Wilkinson microwave anisotropy probe 1-yr data using directional spherical wavelets," Monthly Notices of the Royal Astronomical Society, vol. 359, no. 4, pp. 1583-1596, 2005.

[48] D. Pietrobon, A. Amblard, A. Balbi, P. Cabella, A. Cooray, and D. Marinucci, "Needlet detection of features in the WMAP CMB sky and the impact on anisotropies and hemispherical asymmetries," Physical Review D, vol. 78, no. 10, Article ID 103504, 2008.

[49] Y. Wiaux, P. Vielva, R. B. Barreiro, E. Martínez-González, and P. Vandergheynst, "Non-Gaussianity analysis on local morphological measures of WMAP data," Monthly Notices of the Royal Astronomical Society, vol. 385, no. 2, pp. 939-947, 2008.

[50] A. Curto, E. Martínez-González, and R. B. Barreiro, "Improved constraints on primordial non-gaussianity for the wilkinson microwave anisotropy probe 5-year data," The Astrophysical Journal, vol. 706, no. 1, pp. 399-403, 2009.

[51] Ø. Rudjord, F. K. Hansen, X. Lan, M. Liguori, D. Marinucci, and S. Matarrese, "An estimate of the primordial nongaussianity parameter $\mathrm{f} \mathrm{NL}$ using the needlet bispectrum from wmap," The Astrophysical Journal, vol. 701, no. 1, pp. 369-376, 2009.

[52] P. Vielva, E. Martínez-González, and M. Tucci, "Crosscorrelation of the cosmic microwave background and radio galaxies in real, harmonic and wavelet spaces: detection of the integrated Sachs-Wolfe effect and dark energy constraints," Monthly Notices of the Royal Astronomical Society, vol. 365, no. 3, pp. 891-901, 2006.

[53] D. Pietrobon, A. Balbi, and D. Marinucci, "Integrated SachsWolfe effect from the cross correlation of WMAP 3 year and the NRAO VLA sky survey data: new results and constraints on dark energy," Physical Review D, vol. 74, no. 4, Article ID 043524, 2006.

[54] X. Liu and S. -N. Zhang, "A cross-correlation analysis of WMAP and egret data in wavelet space," The Astrophysical Journal, vol. 636, no. 1, pp. L1-L4, 2006.

[55] J. D. McEwen, Y. Wiaux, M. P. Hobson, P. Vandergheynst, and A. N. Lasenby, "Probing dark energy with steerable wavelets through correlation of WMAP and NVSS local morphological measures," Monthly Notices of the Royal Astronomical Society, vol. 384, no. 4, pp. 1289-1300, 2008.

[56] L. Cao and L.-Z. Fang, "A wavelet-galerkin algorithm of the E/B decomposition of cosmic microwave background polarization maps," The Astrophysical Journal, vol. 706, no. 2, p. 1545, 2009.

[57] D. K. Hammond, Y. Wiaux, and P. Vandergheynst, "Wavelet domain Bayesian denoising of string signal in the cosmic microwave background," Monthly Notices of the Royal Astronomical Society, vol. 398, no. 3, pp. 1317-1332, 2009.

[58] K. Maisinger, M. P. Hobson, and A. N. Lasenby, "Maximumentropy image reconstruction using wavelets," Monthly Notices of the Royal Astronomical Society, vol. 347, no. 1, pp. 339-354, 2004.

[59] Y. Moudden, P. Abbrial, P. Vielva et al., "Independent component separation from incomplete spherical data using wavelets. Application to CMB data analysis," in Proceedings of the International Conference on Physics in Signal and Image Processing (PSIP '05), Toulouse, France, January 2005.

[60] F. K. Hansen, A. J. Banday, H. K. Eriksen, K. M. Górski, and P. B. Lilje, "Foreground subtraction of cosmic microwave background maps using WI-fit (wavelet-based high-resolution fitting of internal templates)," The Astrophysical Journal, vol. 648, no. 2, pp. 784-796, 2006. 
[61] J. Delabrouille, J.-F. Cardoso, M. Le Jeune, M. Betoule, G. Fay, and F. Guilloux, "A full sky, low foreground, high resolution CMB map from WMAP," Astronomy \& Astrophysics, vol. 493, no. 3, pp. 835-837, 2009.

[62] J. L. Sanz, F. Argüeso, L. Cayón, E. Martínez-González, R. B. Barreiro, and L. Toffolatti, "Wavelets applied to cosmic microwave background maps: a multiresolution analysis for denoising," Monthly Notices of the Royal Astronomical Society, vol. 309, no. 3, pp. 672-680, 1999.

[63] G. Faÿ, F. Guilloux, M. Betoule, J.-F. Cardoso, J. Delabrouille, and $\mathrm{M}$. Le Jeune, "CMB power spectrum estimation using wavelets," Physical Review D, vol. 78, no. 8, Article ID 083013, 2008.

[64] P. Mukherjee and Y. Wang, "Model-independent reconstruction of the primordial power spectrum from Wilkinson microwave anistropy probe data," The Astrophysical Journal, vol. 599, no. 1, pp. 1-6, 2003.

[65] J. D. McEwen, P. Vielva, Y. Wiaux et al., "Cosmological applications of a wavelet analysis on the sphere," Journal of Fourier Analysis and Applications, vol. 13, no. 4, pp. 495-510, 2007.

[66] P. Vielva, "Probing the Gaussianity and the statistical isotropy of the CMB with spherical wavelets," in Wavelets XII, D. van de Ville, V. K. Goyal, and M. Papadakis, Eds., vol. 6701 of Proceedings of the SPIE, p. 19, San Diego, Calif, USA, 2007.

[67] R. T. Odgen, Essential Wavelets for Statistical Applications and Data Analysis, Birkhauser, Boston, Mass, USA, 1997.

[68] J.-P. Antoine, R. Murenzi, P. Vandergheynst, and S. T. Ali, Two-Dimensional Wavelets and Their Relatives, Cambridge University Press, Cambridge, UK, 2004.

[69] J.-P. Antoine and P. Vandergheynst, "Wavelets on the nsphere and related manifolds," Journal of Mathematical Physics, vol. 39, no. 8, pp. 3987-4008, 1998.

[70] Y. Wiaux, L. Jacques, and P. Vandergheynst, "Correspondence principle between spherical and euclidean wavelets," The Astrophysical Journal, vol. 632, no. 1, pp. 15-28, 2005.

[71] J. L. Sanz, D. Herranz, M. López-Caniego, and F. Argüeso, "Wavelets on the sphere. Application to the detection problem," in Proceedings of the 14th European Signal Processing Conference (EUSIPCO '06), F. Gini and E. E. Kuruoglu, Eds., Florence, Italy, September 2006.

[72] Y. Wiaux, J. D. McEwen, and P. Vielva, "Complex data processing: fast wavelet analysis on the sphere," Journal of Fourier Analysis and Applications, vol. 13, no. 4, pp. 477-493, 2007.

[73] M. Górski, E. Hivon, A. J. Banday et al., "HEALPix: a framework for high-resolution discretization and fast analysis of data distributed on the sphere," The Astrophysical Journal, vol. 622, no. 2, pp. 759-771, 2005.

[74] C.-G. Park, "Non-Gaussian signatures in the temperature fluctuation observed by the Wilkinson microwave anisotropy probe," Monthly Notices of the Royal Astronomical Society, vol. 349, no. 1, pp. 313-320, 2004.

[75] E. L. Wright, X. Chen, N. Odegard et al., "Five-year wilkinson microwave anisotropy probe observations: source catalog," Astrophysical Journal, Supplement Series, vol. 180, no. 2, pp. 283-295, 2009.

[76] M. Cruz, L. Cayón, E. Martínez-González, P. Vielva, and J. Jin, "The non-gaussian cold spot in the 3 year Wilkinson microwave anisotropy probe data," The Astrophysical Journal, vol. 655, no. 1, pp. 11-20, 2007.

[77] P. Coles, "Statistical geometry and the microwave background," Monthly Notices of the Royal Astronomical Society, vol. 234, pp. 509-531, 1988.
[78] J. R. Gott III, C. Park, R. Juszkiewicz et al., "Topology of microwave background fluctuations: theory," The Astrophysical Journal, vol. 352, no. 1, pp. 1-14, 1990.

[79] J. Schmalzing and K. M. Górski, "Minkowski functionals used in the morphological analysis of cosmic microwave background anisotropy maps," Monthly Notices of the Royal Astronomical Society, vol. 297, no. 2, pp. 355-365, 1998.

[80] D. Donoho and J. Jin, "Higher criticism for detecting sparse heterogeneous mixtures," Annals of Statistics, vol. 32, no. 3, pp. 962-994, 2004.

[81] L. Cayón, J. Jin, and A. Treaster, "Higher criticism statistic: detecting and identifying non-Gaussianity in the WMAP first-year data," Monthly Notices of the Royal Astronomical Society, vol. 362, no. 3, pp. 826-832, 2005.

[82] M. Cruz, M. Tucci, E. Martínez-González, and P. Vielva, "The non-Gaussian cold spot in Wilkinson microwave anisotropy probe: significance, morphology and foreground contribution," Monthly Notices of the Royal Astronomical Society, vol. 369, no. 1, pp. 57-67, 2006.

[83] R. Zhang and D. Huterer, "Disks in the sky: a reassessment of the WMAP "cold spot"', Astroparticle Physics, vol. 33, no. 2, pp. 69-74, 2010.

[84] D. Herranz, J. L. Sanz, R. B. Barreiro, and E. MartínezGonzález, "Scale-adaptive filters for the detection/separation of compact sources," The Astrophysical Journal, vol. 580, no. 1, pp. 610-625, 2002.

[85] M. P. Hobson, A. W. Jones, and A. N. Lasenby, "Wavelet analysis and the detection of non-Gaussianity in the cosmic microwave background," Monthly Notices of the Royal Astronomical Society, vol. 309, no. 1, pp. 125-140, 1999.

[86] C. Räth, P. Schuecker, and A. J. Banday, "A scaling index analysis of the Wilkinson microwave anisotropy probe threeyear data: signatures of non-Gaussianities and asymmetries in the cosmic microwave background," Monthly Notices of the Royal Astronomical Society, vol. 380, no. 2, pp. 466-478, 2007.

[87] G. Rossmanith, C. Räth, A. J. Banday, and G. Morfill, "Nongaussian signatures in the five-year WMAP data as identified with isotropic scaling indices," Monthly Notices of the Royal Astronomical Society, vol. 399, no. 4, pp. 1921-1933, 2009.

[88] V. G. Gurzadyan, A. E. Allahverdyan, T. Ghahramanyan et al., "Kolmogorov cosmic microwave background sky," Astronomy \& Astrophysics, vol. 497, no. 2, pp. 343-346, 2009.

[89] M. Tegmark, A. de Oliveira-Costa, and A. J.S. Hamilton, "High resolution foreground cleaned CMB map from WMAP,” Physical Review D, vol. 68, no. 12, Article ID 123523, 2003.

[90] J. L. Jonas, E. E. Baart, and G. D. Nicolson, "The Rhodes/HartRAO 2326-MHz radio continuum survey," Monthly Notices of the Royal Astronomical Society, vol. 297, no. 4, pp. 977-989, 1998.

[91] D. P. Finkbeiner, "A full-sky $\mathrm{H} \alpha$ template for microwave foreground prediction," Astrophysical Journal, Supplement Series, vol. 146, no. 2, pp. 407-415, 2003.

[92] D. P. Finkbeiner, M. Davis, and D. J. Schlegel, "Extrapolation of galactic dust emission at 100 microns to cosmic microwave background radiation frequencies using FIRAS," The Astrophysical Journal, vol. 524, no. 2, pp. 867-886, 1999.

[93] R. A. Sunyaev and Ya. B. Zeldovich, "Small-scale fluctuations of relic radiation," Astrophysics and Space Science, vol. 7, no. 1, pp. 3-19, 1970.

[94] S. Brough, D. A. Forbes, V. A. Kilborn, W. Couch, and M. Colless, "Eridanus-a supergroup in the local Universe?" Monthly Notices of the Royal Astronomical Society, vol. 369, no. 3, pp. 1351-1374, 2006. 
[95] M. Cruz, E. Martínez-González, P. Vielva, J. M. Diego, M. Hobson, and N. Turok, "The CMB cold spot: texture, cluster or void?" Monthly Notices of the Royal Astronomical Society, vol. 390, no. 3, pp. 913-919, 2008.

[96] E. Martínez-González and J. L. Sanz, "CMB anisotropies generated by cosmic voids and great attractors," Monthly Notices of the Royal Astronomical Society, vol. 247, no. 3, pp. 473-478, 1990.

[97] E. Martínez-González, J. L. Sanz, and J. Silk, "Anisotropies in the microwave sky due to nonlinear structures," The Astrophysical Journal, vol. 355, no. 1, pp. L5-L9, 1990.

[98] K. T. Indue and J. Silk, "Local voids as the origin of largeangle cosmic microwave background anomalies. I," The Astrophysical Journal, vol. 648, no. 1, pp. 23-30, 2006.

[99] K. T. Inoue and J. Silk, "Local voids as the origin of largeangle cosmic microwave background anomalies: the effect of a cosmological constant," The Astrophysical Journal, vol. 664, no. 2, pp. 650-659, 2007.

[100] L. Rudnick, S. Brown, and L. R. Williams, "Extragalactic radio sources and the WMAP cold spot," The Astrophysical Journal, vol. 671, no. 1, pp. 40-44, 2007.

[101] J. J. Condon, W. D. Cotton, E. W. Greisen et al., "The NRAO VLA sky survey," Astronomical Journal, vol. 115, no. 5, pp. 1693-1716, 1998.

[102] J. M. Colberg, R. K. Sheth, A. Diaferio, L. Gao, and N. Yoshida, "Voids in a ACDM universe," Monthly Notices of the Royal Astronomical Society, vol. 360, no. 1, pp. 216-226, 2005.

[103] K. M. Smith and D. Huterer, "No evidence for the cold spot in the NVSS radio survey," Monthly Notices of the Royal Astronomical Society, vol. 403, no. 1, pp. 2-8, 2010.

[104] B. R. Granett, I. Szapudi, and M. C. Neyrinck, "Galaxy counts on the cosmic microwave background cold spot," The Astrophysical Journal, vol. 714, no. 1, p. 825, 2010.

[105] M. N. Bremer, J. Silk, L. J. M. Davies, and M. D. Lehnert, "A redshift survey towards the cosmic microwave background cold spot," Monthly Notices of the Royal Astronomical Society, vol. 404, no. 1, pp. L69-L73, 2010.

[106] M. Cruz, N. Turok, P. Vielva, E. Martínez-González, and M. Hobson, "A cosmic microwave background feature consistent with a cosmic texture," Science, vol. 318, no. 5856, pp. 1612 1614, 2007.

[107] N. Turok, "Global texture as the origin of cosmic structure," Physical Review Letters, vol. 63, no. 24, pp. 2625-2628, 1989.

[108] A. Vilenkin and E. P. S. Shellard, Cosmic Strings and Other Topological Defects, Cambridge University Press, Cambridge, UK, 2000.

[109] N. Turok and D. Spergel, "Global texture and the microwave background," Physical Review Letters, vol. 64, no. 23, pp. 2736-2739, 1990.

[110] A. Silvestri and M. Trodden, "Non-Gaussian signatures from the postinflationary early Universe," Physical Review Letters, vol. 103, no. 25, Article ID 251301, 2009.

[111] N. Bevis, M. Hindmarsh, and M. Kunz, "WMAP constraints on inflationary models with global defects," Physical Review D, vol. 70, no. 4, Article ID 043508, 9 pages, 2004.

[112] J. Urrestilla, N. Bevis, M. Hindmarsh, M. Kunz, and A. R. Liddle, "Cosmic microwave anisotropies from BPS semilocal strings," Journal of Cosmology and Astroparticle Physics, vol. 2008, no. 7, article 010, 2008.

[113] E. Komatsu, K. M. Smith, J. Dunkley et al., "Seven-year Wilkinson microwave anisotropy probe (WMAP) observations: cosmological interpretation," http://arxiv.org/abs/ 1001.4538 .
[114] U.-L. Pen, D. N. Spergel, and N. Turok, "Cosmic structure formation and microwave anisotropies from global field ordering," Physical Review D, vol. 49, no. 2, pp. 692-729, 1994.

[115] R. Durrer, M. Kunz, and A. Melchiorri, "Cosmic microwave background anisotropies from scaling seeds: global defect models," Physical Review D, vol. 59, no. 12, Article ID 123005, 26 pages, 1999.

[116] P. Mukherjee, D. Parkinson, and A. R. Liddle, "A nested sampling algorithm for cosmological model selection," The Astrophysical Journal, vol. 638, no. 2, pp. L51-L54, 2006.

[117] M. Bridges, J. D. McEwen, A. N. Lasenby, and M. P. Hobson, "Markov chain Monte Carlo analysis of Bianchi VIIh models," Monthly Notices of the Royal Astronomical Society, vol. 377, no. 4, pp. 1473-1480, 2007.

[118] P. Mukherjee and A. R. Liddle, "Planck and re-ionization history: a model selection view," Monthly Notices of the Royal Astronomical Society, vol. 389, no. 1, pp. 231-236, 2008.

[119] P. Carvalho, G. Rocha, and M. P. Hobson, "A fast Bayesian approach to discrete object detection in astronomical data sets-PowellSnakes I," Monthly Notices of the Royal Astronomical Society, vol. 393, no. 3, pp. 681-702, 2009.

[120] P. Vielva and J. L. Sanz, "Analysis of non-Gaussian cosmic microwave background maps based on the N-pdf. Application to Wilkinson microwave anisotropy probe data," Monthly Notices of the Royal Astronomical Society, vol. 397, no. 2, pp. 837-848, 2009.

[121] H. Jeffreys, Theory of Probability, Oxford University Press, Oxford, UK, 3rd edition, 1961.

[122] M. Bridges, J. D. McEwen, M. Cruz et al., "Bianchi VIIh models and the cold spot texture," Monthly Notices of the Royal Astronomical Society, vol. 390, no. 4, pp. 1372-1376, 2008.

[123] T. R. Jaffe, A. J. Banday, H. K. Eriksen, K. M. Górski, and F. K. Hansen, "Evidence of vorticity and shear at large angular scales in the WMAP data: a violation of cosmological isotropy?" The Astrophysical Journal, vol. 629, no. 1, pp. L1L4, 2005.

[124] D. N. Spergel, N. Turok, W. H. Press, and B. S. Ryden, "Global texture as the origin of large-scale structure: numerical simulations of evolution," Physical Review D, vol. 43, no. 4, pp. 1038-1046, 1991.

[125] F. Feroz, M. P. Hobson, and M. Bridges, "MultiNest: an efficient and robust Bayesian inference tool for cosmology and particle physics," Monthly Notices of the Royal Astronomical Society, vol. 398, no. 4, pp. 1601-1614, 2009.

[126] P. Vielva, E. Martínez-González, M. Cruz, R. B. Barreiro, and M. Tucci, "CMB polarization as a probe of the anomalous nature of the Cold Spot," Monthly Notices of the Royal Astronomical Society, http://fr.arxiv.org/abs/1002.4029. In press.

[127] G. Cowan, Statistical Data Analysis, Oxford University Press, Oxford, UK, 1998.

[128] J. Tauber et al., "Planck pre-launch status: The Planck mission," Astronomy \& Astrophysics, Vol. 520, id.A1.

[129] J. A. Rubiño-Martín, R. Rebolo, M. Tucci et al., "The Quijote CMB experiment," in Highlights of Spanish Astrophysics V, Proceedings of the VIII Scientific Meeting of the Spanish Astronomical Society (SEA), J. Gorgas, L. J. Goicoechea, J. I. Gonzalez-Serrano, and J. M. Diego, Eds., pp. 127-135, Santander, spain, July 2010.

[130] S. Das and D. N. Spergel, "CMB lensing and the WMAP cold spot," Physical Review D, vol. 79, no. 4, Article ID 043007, 2009. 

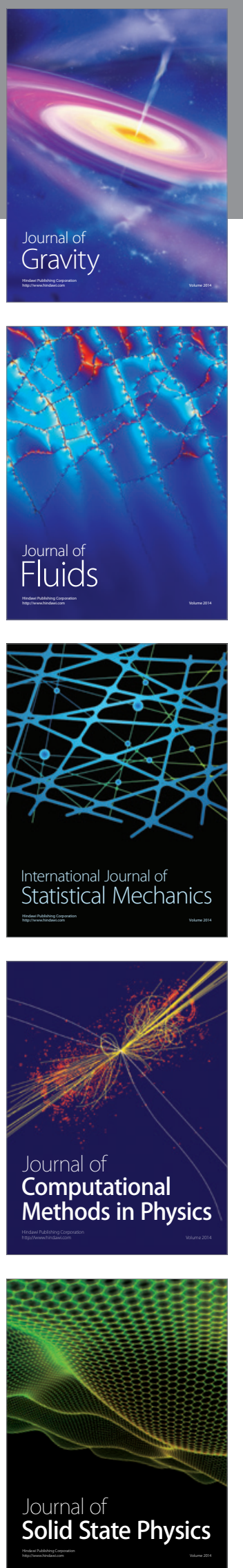

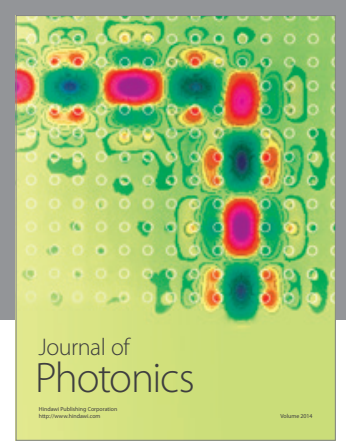

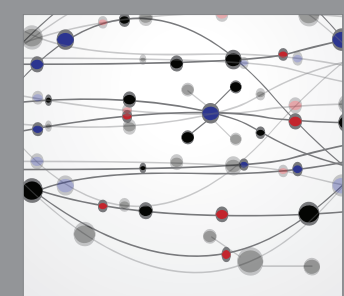

The Scientific World Journal
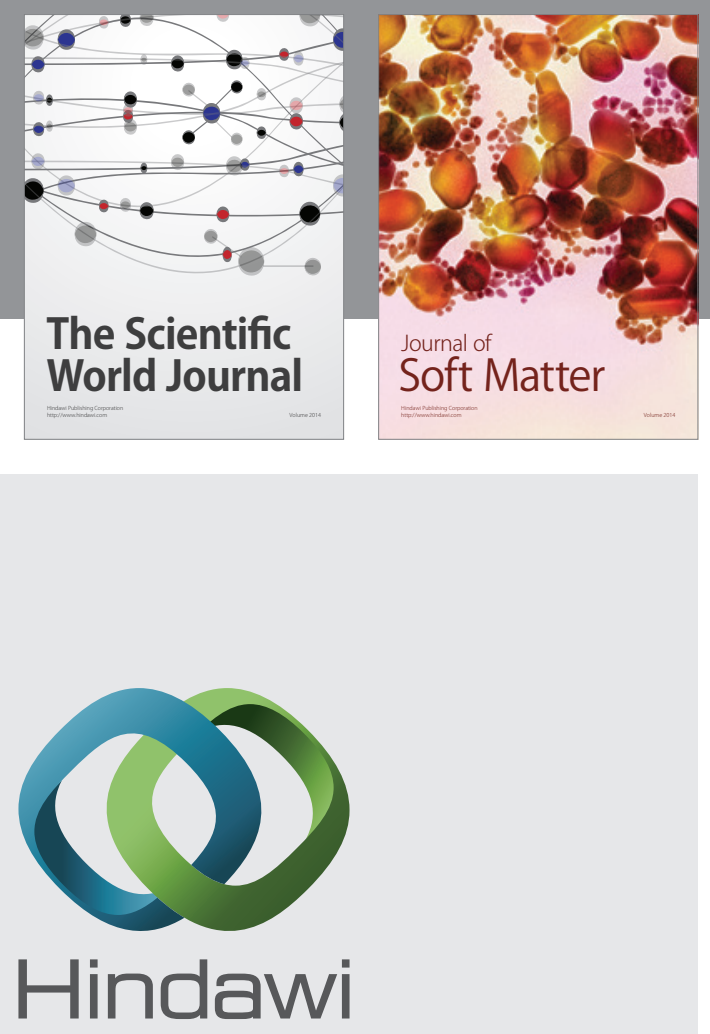

Submit your manuscripts at

http://www.hindawi.com
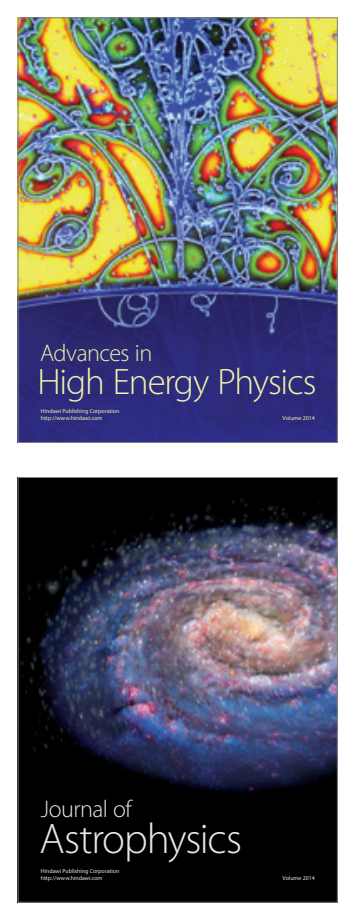
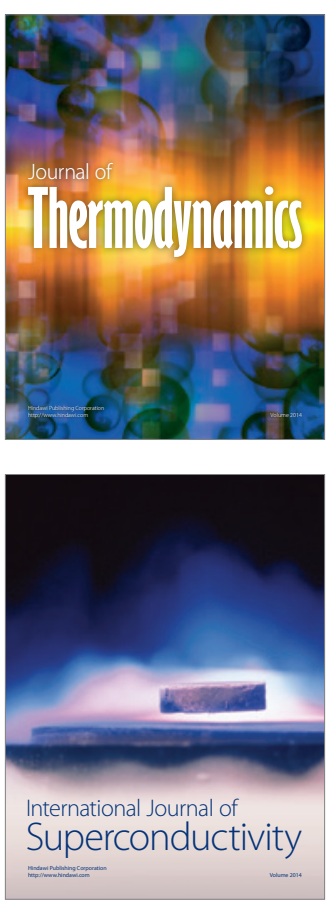
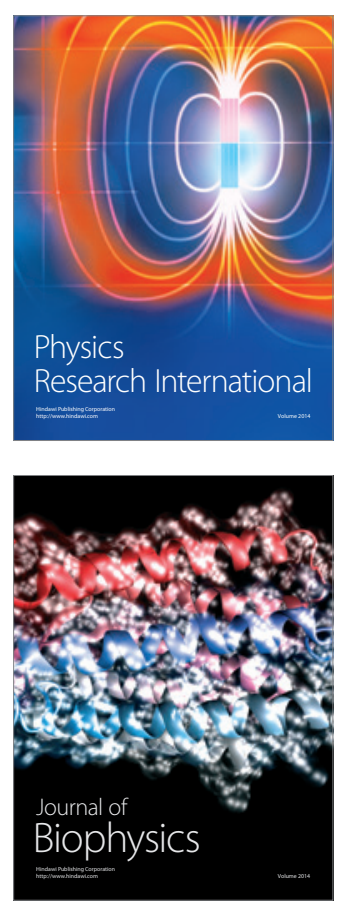
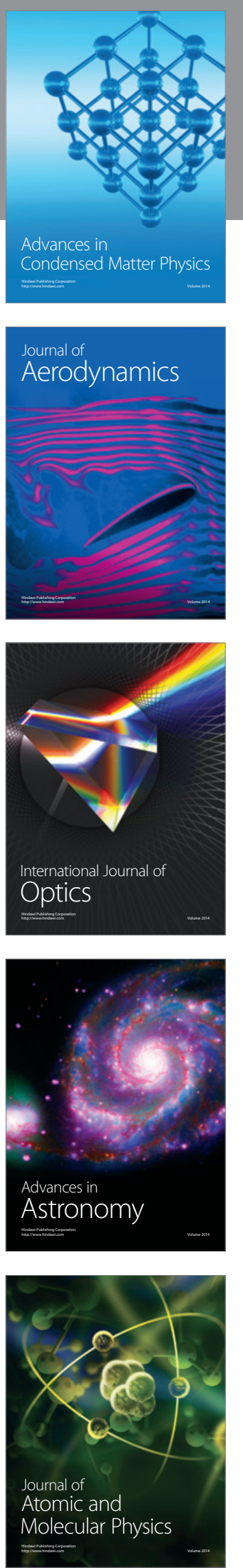